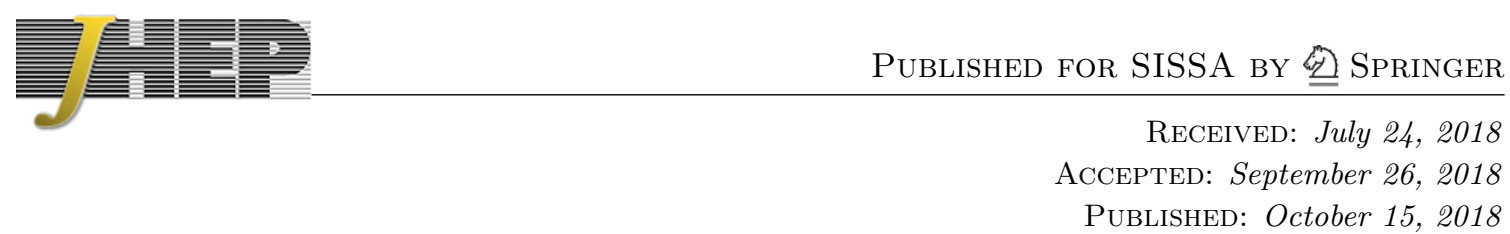

\title{
Vertex operators for the plane wave pure spinor string
}

\author{
Osvaldo Chandia ${ }^{a}$ and Brenno Carlini Vallilo ${ }^{b}$ \\ ${ }^{a}$ Departamento de Ciencias, Facultad de Artes Liberales 6 UAI Physics Center, \\ Universidad Adolfo Ibáñez, \\ Diagonal Las Torres 2640, Santiago, Chile \\ ${ }^{b}$ Departamento de Ciencias Físicas, Universidad Andres Bello, \\ Sazie 2212, Santiago, Chile \\ E-mail: ochandiaq@gmail.com, vallilo@gmail.com
}

ABSTRACT: In this work we give an explicit construction for the vertex operators of massless states in the pure spinor superstring in a plane wave background. The construction is based on the observation that the full action can be divided in two parts, where the simpler one is based on a smaller coset and closely resembles the gauge fixed Green-Schwarz action.

KEYwords: AdS-CFT Correspondence, Conformal Field Models in String Theory, Supergravity Models, Superspaces

ARXiv EPrint: 1807.05149 


\section{Contents}

1 Introduction 1

2 BMN limit of the $\mathfrak{p s u}(2,2 \mid 4)$ algebra $\quad 2$

2.1 Organizing in terms of $\mathfrak{p s u}(2 \mid 2) \oplus \mathfrak{p s u}(2 \mid 2) \oplus \mathbb{R}^{2}$

2.2 Casimir of the contracted algebra and spectrum 5

$\begin{array}{llr}3 & \text { Supergeometry } & 6\end{array}$

4 Action, BRST and conformal invariance $\quad 9$

5 Massless vertex operators 13

$\begin{array}{llr}6 & \text { Integrated vertex operator } & 18\end{array}$

$\begin{array}{ll}\text { A Conventions } & 24\end{array}$

B Open and flat $\quad 25$

\section{Introduction}

For more than ten years there has been enormous progress in the understanding of both sides of the AdS/CFT conjecture due to the presence of integrable structures [1,2]. Even after all the progress it is still not known how to obtain the physical spectrum and amplitudes at finite AdS radius.

In principle one could use perturbation theory at large radius using the pure spinor description for the superstring. Some partial results have been obtained for the massless spectrum [3-5], but a complete dictionary between BPS states and the corresponding vertex operator is still missing. An attempt to describe a massive state was made in [6], however some contributions were incorrectly ignored [7]. Although the pure spinor sigma model is classically integrable $[8,9]$ and some quantum aspects have been studied $[10,11]$ there are no techniques available to help computing the spectrum. It is possible that the formalism developed in [12] and applied in [13, 14] could be generalized to the case of AdS pure spinor string.

Before attacking the AdS case we could first look at the simpler BMN limit [15]. The Green-Schwarz superstring in this space was studied extensively $[16,17]$ and pure spinor string was studied in [18] and [19]. The approach of [19] was to use the background field expansion of the usual $A d S_{5} \times S^{5}$ pure spinor string around the BPS state with $\mathrm{E}=\mathrm{J}$. Although the resulting model was free and the full spectrum can be computed, the 
isometries of the plane wave background were not manifest in this approach and the BMN limit spontaneously breaks conformal invariance. The latter severely reduces its usefulness.

In this paper we will study massless vertex operators for the string defined in [18]. Although the sigma model is not as simple as the free gauge fixed Green-Schwarz version, its structure simplifies some computations. For example, it was proved in [18] that the beta function has only a vanishing one loop contribution. We will find an explicit construction for the unintegrated vertex operator using the isometries of the background. As was shown in [18] the sigma model action can be divided in a simpler action depending only on the coordinates of a reduced superspace and a more complicated part. Our construction starts by first fixing the form of the vertex operator depending only on the variables of this reduced superspace using the BRST condition. In order to fix its full form we will use a Virasorolike condition coming the Casimir operator of the isometry algebra. It is important to note the for the flat space case, the physical massless vertex operator can be fixed by using only half of the superspace and the Virasoro condition. We then compute the integrated vertex operator using the standard BRST descent procedure. Although the final expression is not very illuminating, the plane wave background admits D9-branes and the open string version is simple and could lead to the construction of DDF-like operators [20].

This paper is organized as follows. In section 2 we review how to obtain the isometry algebra of the plane wave limit of $A d S_{5} \times S^{5}$ background as a contraction of the $\mathfrak{p s u}(2,2 \mid 4)$ algebra. In section 3 we describe the supergeometry of the plane wave background and compute the covariant derivatives and symmetry generators. The pure spinor description of the superstring in BMN limit of $A d S_{5} \times S^{5}$ is reviewed in section 4. In section 5 we construct the unintegrated vertex operator for all BPS states using part of the BRST charge and the isometries of the background. Finally in section 6 we use the standard descent procedure to find the general form of the integrated vertex operator. The appendices contain conventions and some details left out from the body of the paper.

\section{BMN limit of the $\mathfrak{p s u}(2,2 \mid 4)$ algebra}

The isometry algebra of the BMN limit [15] of $A d S_{5} \times S^{5}$ is obtained as a contraction of the $\mathfrak{p s u}(2,2 \mid 4)$ algebra [21], which can be understood geometrically as the Penrose limit of the original AdS space $[22,23]$. The idea is to look for the isometries a massless particle with very high energy in $A d S_{5}$ and very high angular momentum in $S^{5}$ sees. Let us first look at the bosonic subalgebra generated by $\left(\mathrm{M}_{A B}, \mathrm{M}_{A}, \mathrm{P}_{A}, \mathrm{~T}, \mathrm{M}_{I J}, \mathrm{M}_{I}, \mathrm{P}_{I}, \mathrm{~J}\right)$ which is $\mathfrak{s o}(2,4) \oplus \mathfrak{s o}(6)$ in an $\mathfrak{s o}(4) \oplus \mathfrak{s o}(4)$ basis. Their commutators can be found in the appendix A. We chose a convention where the translations $\left(\mathrm{P}_{A}, \mathrm{~T}, \mathrm{P}_{I}, \mathrm{~J}\right)$ are hermitian and the rotations $\left(\mathrm{M}_{A B}, \mathrm{M}_{A}, \mathrm{M}_{I J}, \mathrm{M}_{I}\right)$ are anti-hermitian. Note that in the limit $R \rightarrow \infty$ the algebra defined by these generators is $\mathfrak{i s o}(1,4) \oplus \mathfrak{i s o}(5)$. This is expected to be enhanced to the full $\mathfrak{i s o}(1,9)$ since new conserved currents will appear as $R \rightarrow \infty$.

Since the particle is massless the eigenvalues of $E$ and $J$ should be the same. So in order to have a generator the survives this limit we define

$$
\mathrm{E}_{+}=R(\mathrm{~T}+\mathrm{J}) .
$$


Furthermore, since their sum will diverge we define

$$
\mathrm{E}_{-}=R^{-1}(\mathrm{~T}-\mathrm{J})
$$

where $R$ is the radius of $A d S_{5}$ and $S^{5}$. Both of these generators will be well defined in the limit $R \rightarrow \infty$. Now we invert these definitions

$$
\mathrm{T}=\frac{1}{2}\left(R \mathrm{E}_{-}+R^{-1} \mathrm{E}_{+}\right), \quad \mathrm{J}=\frac{1}{2}\left(R^{-1} \mathrm{E}_{+}-R \mathrm{E}_{-}\right) .
$$

Boosts in the $\mathrm{T}$ and $\mathrm{J}$ directions $\mathrm{M}_{A}$ and $\mathrm{M}_{I}$ also have to be re-scaled

$$
\mathrm{M}_{A}=-\mathrm{i} R \overline{\mathrm{P}}_{A}, \quad \mathrm{M}_{I}=\mathrm{i} R \overline{\mathrm{P}}_{I},
$$

now $\overline{\mathrm{P}}_{A}$ and $\overline{\mathrm{P}}_{I}$ are hermitian. The reason for this notation will become clear later; we will be able to organize the generators in representations of $\mathfrak{s u}(2 \mid 2) \oplus \mathfrak{s u}(2 \mid 2)$.

Using these definitions and the commutators in appendix A, after taking the $R \rightarrow \infty$ limit, the non-vanishing commutators are (besides the $\mathfrak{s o}(4) \oplus \mathfrak{s o}(4)$ algebra generated by $\mathrm{M}_{A B}$ and $\mathrm{M}_{I J}$, which is left unchanged):

$$
\begin{array}{rlrl}
{\left[\mathrm{P}_{A}, \overline{\mathrm{P}}_{B}\right]} & =-\frac{\mathrm{i}}{2} \delta_{A B} \mathrm{E}_{-}, & {\left[\mathrm{P}_{I}, \overline{\mathrm{P}}_{J}\right]=-\frac{\mathrm{i}}{2} \delta_{I J} \mathrm{E}_{-}} \\
{\left[\mathrm{E}_{+}, \mathrm{P}_{A}\right]=\mathrm{i} \overline{\mathrm{P}}_{A},} & {\left[\mathrm{E}_{+}, \overline{\mathrm{P}}_{A}\right]=-\mathrm{iP}_{A},} \\
{\left[\mathrm{E}_{+}, \mathrm{P}_{I}\right]=\mathrm{i} \overline{\mathrm{P}}_{I},} & {\left[\mathrm{E}_{+}, \overline{\mathrm{P}}_{I}\right]=-\mathrm{iP}_{I} .}
\end{array}
$$

We can see that the generators $\left(\mathrm{P}_{A}, \overline{\mathrm{P}}_{A}, \mathrm{P}_{I}, \overline{\mathrm{P}}_{I}, \mathrm{E}_{-}\right)$form a Heisenberg algebra $\mathfrak{h}(8)$ with central element $\mathrm{E}_{-}$and $\mathrm{E}_{+}$acts as an outer automorphism of the algebra that commutes with $\mathfrak{s o}(4) \oplus \mathfrak{s o}(4)$. It should be stressed that the $\mathfrak{s o}(4) \oplus \mathfrak{s o}(4)$ algebra is not promoted to full $\mathfrak{s o}(8)$ in the limit $R \rightarrow \infty$. The reason for this is the presence of the supercharges, which we will now describe.

We will first define new scalings for the supercharges $\left(\mathrm{Q}_{a}, \mathrm{Q}_{\dot{a}}, \overline{\mathrm{Q}}_{a}, \overline{\mathrm{Q}}_{\dot{a}}\right)$. We will try

$$
\mathrm{S}_{a}=R^{\frac{1}{2}} \mathrm{Q}_{a} . \quad \overline{\mathrm{S}}_{a}=R^{\frac{1}{2}} \overline{\mathrm{Q}}_{a}, \quad \mathrm{~S}_{\dot{a}}=R^{-\frac{1}{2}} \mathrm{Q}_{\dot{a}}, \quad \overline{\mathrm{S}}_{\dot{a}}=R^{-\frac{1}{2}} \overline{\mathrm{Q}}_{\dot{a}} .
$$

With these definitions we obtain the expected supersymmetry algebra

$$
\begin{aligned}
& \left\{\mathrm{S}_{a}, \mathrm{~S}_{b}\right\}=\left\{\overline{\mathrm{S}}_{a}, \overline{\mathrm{S}}_{b}\right\}=\delta_{a b} \mathrm{E}_{+}, \quad\left\{\mathrm{S}_{\dot{a}}, \mathrm{~S}_{\dot{b}}\right\}=\left\{\overline{\mathrm{S}}_{\dot{a}}, \overline{\mathrm{S}}_{\dot{b}}\right\}=\delta_{\dot{a} \dot{b}} \mathrm{E}_{-}, \\
& \left\{\mathrm{S}_{a}, \mathrm{~S}_{\dot{a}}\right\}=\left\{\overline{\mathrm{S}}_{a}, \overline{\mathrm{S}}_{\dot{a}}\right\}=\sigma_{a \dot{a}}^{A} \mathrm{P}_{A}+\sigma_{a \dot{a}}^{I} \mathrm{P}_{I},
\end{aligned}
$$

with $\mathrm{E}_{-}$still playing the role of a central charge. Also note that $\left(\mathrm{S}_{a}, \mathrm{~S}_{\dot{a}}, \overline{\mathrm{S}}_{a}, \overline{\mathrm{S}}_{\dot{a}}\right)$ are still $\mathfrak{s o}(4) \oplus \mathfrak{s o}(4)$ spinors and have the expected commutators with the generators $\left(\mathrm{M}_{A B}, \mathrm{M}_{I J}\right)$. Furthermore, with (2.8) and taking the $R \rightarrow \infty$ limit we obtain

$$
\begin{aligned}
& {\left[\mathrm{E}_{+}, \mathrm{S}_{a}\right]=\left[\mathrm{E}_{+}, \overline{\mathrm{S}}_{a}\right]=0,} \\
& {\left[\mathrm{P}_{A}, \mathrm{~S}_{\dot{a}}\right]=\left[\mathrm{P}_{A}, \overline{\mathrm{S}}_{\dot{a}}\right]=\left[\overline{\mathrm{P}}_{A}, \mathrm{~S}_{\dot{a}}\right]=\left[\overline{\mathrm{P}}_{A}, \overline{\mathrm{S}}_{\dot{a}}\right]=0,} \\
& {\left[\mathrm{P}_{I}, \mathrm{~S}_{\dot{a}}\right]=\left[\mathrm{P}_{I}, \overline{\mathrm{S}}_{\dot{a}}\right]=\left[\overline{\mathrm{P}}_{I}, \mathrm{~S}_{\dot{a}}\right]=\left[\overline{\mathrm{P}}_{I}, \overline{\mathrm{S}}_{\dot{a}}\right]=0,} \\
& {\left[\mathrm{E}_{+}, \mathrm{S}_{\dot{a}}\right]=\mathrm{i} \Pi_{\dot{a} \dot{b}} \overline{\mathrm{S}}_{\dot{b}}, \quad\left[\mathrm{E}_{+}, \overline{\mathrm{S}}_{\dot{a}}\right]=-\mathrm{i} \Pi_{\dot{a} \dot{b}} \mathrm{~S}_{\dot{b}},}
\end{aligned}
$$




$$
\begin{aligned}
{\left[\mathrm{P}_{A}, \mathrm{~S}_{a}\right] } & =-\frac{\mathrm{i}}{2}\left(\sigma_{A} \Pi\right)_{a \dot{a}} \overline{\mathrm{S}}_{\dot{a}}, & {\left[\mathrm{P}_{A}, \overline{\mathrm{S}}_{a}\right] } & =\frac{\mathrm{i}}{2}\left(\sigma_{A} \Pi\right)_{a \dot{a}} \mathrm{~S}_{\dot{a}} \\
{\left[\mathrm{P}_{I}, \mathrm{~S}_{a}\right] } & =-\frac{\mathrm{i}}{2}\left(\sigma_{I} \Pi\right)_{a \dot{a}} \overline{\mathrm{S}}_{\dot{a}}, & {\left[\mathrm{P}_{I}, \overline{\mathrm{S}}_{a}\right] } & =\frac{\mathrm{i}}{2}\left(\sigma_{I} \Pi\right)_{a \dot{a}} \mathrm{~S}_{\dot{a}} \\
{\left[\overline{\mathrm{P}}_{A}, \mathrm{~S}_{a}\right] } & =\frac{\mathrm{i}}{2}\left(\sigma_{A}\right)_{a \dot{a}} \mathrm{~S}_{\dot{a}}, & {\left[\overline{\mathrm{P}}_{A}, \overline{\mathrm{S}}_{a}\right] } & =\frac{\mathrm{i}}{2}\left(\sigma_{A}\right)_{a \dot{a}} \overline{\mathrm{S}}_{\dot{a}}, \\
{\left[\overline{\mathrm{P}}_{I}, \mathrm{~S}_{a}\right] } & =\frac{\mathrm{i}}{2}\left(\sigma_{I}\right)_{a \dot{a}} \mathrm{~S}_{\dot{a}}, & {\left[\overline{\mathrm{P}}_{I}, \overline{\mathrm{S}}_{a}\right] } & =\frac{\mathrm{i}}{2}\left(\sigma_{I}\right)_{a \dot{a}} \overline{\mathrm{S}}_{\dot{a}},
\end{aligned}
$$

and finally we have that

$$
\begin{aligned}
& \left\{\mathrm{S}_{\dot{a}}, \overline{\mathrm{S}}_{\dot{b}}\right\}=0 \\
& \left\{\mathrm{~S}_{a}, \overline{\mathrm{S}}_{b}\right\}=-\frac{\mathrm{i}}{2}\left(\left(\sigma^{A B} \Pi\right)_{a b} \mathrm{M}_{A B}-\left(\sigma^{I J} \Pi\right)_{a b} \mathrm{M}_{I J}\right), \\
& \left\{\mathrm{S}_{a}, \overline{\mathrm{S}}_{\dot{b}}\right\}=\left(\sigma^{A} \Pi\right)_{a \dot{b}} \overline{\mathrm{P}}_{A}+\left(\sigma^{I} \Pi\right)_{a \dot{b}} \overline{\mathrm{P}}_{I}, \\
& \left\{\mathrm{~S}_{\dot{a}}, \overline{\mathrm{S}}_{a}\right\}=-\left(\sigma^{A} \Pi\right)_{a \dot{a}} \overline{\mathrm{P}}_{A}-\left(\sigma^{I} \Pi\right)_{a \dot{a}} \overline{\mathrm{P}}_{I},
\end{aligned}
$$

where $\left(\sigma^{i} \Pi\right)$ always means $\left(\sigma^{i}\right)_{a \dot{b}} \Pi_{\dot{b} \dot{a}}$ and $\Pi$ is symmetric, traceless and squares to identity. This concludes the contraction of the $\mathfrak{p s u}(2,2 \mid 4)$ algebra.

\subsection{Organizing in terms of $\mathfrak{p s u}(2 \mid 2) \oplus \mathfrak{p s u}(2 \mid 2) \oplus \mathbb{R}^{2}$}

We can see that $\mathrm{E}_{+}$acts as a rotation operator for $\left(\mathrm{P}_{A}, \mathrm{P}_{I}, \mathrm{~S}_{\dot{a}}\right) \times\left(\overline{\mathrm{P}}_{A}, \overline{\mathrm{P}}_{I}, \overline{\mathrm{S}}_{\dot{a}}\right)$. Furthermore, the generators $\left(\mathrm{M}_{A B}, \mathrm{M}_{I J}\right)$ also rotates this set. It turns out that the we can organize all generators in a Jordan structure $[24] \mathfrak{l}^{-} \oplus \mathfrak{l}^{0} \oplus \mathfrak{l}^{+}$that satisfies $^{1}$

$$
\begin{aligned}
{\left[\mathfrak{l}^{0}, \mathfrak{l}^{0}\right] } & \subset \mathfrak{l}^{0}, \quad\left[\mathfrak{l}^{0}, \mathfrak{l}^{-}\right] \subset \mathfrak{l}^{-}, \quad\left[\mathfrak{l}^{0}, \mathfrak{l}^{+}\right] \subset \mathfrak{l}^{+}, \quad\left[\mathfrak{l}^{+}, \mathfrak{l}^{-}\right] \subset \mathfrak{l}^{0}, \\
{\left[\mathfrak{l}^{+}, \mathfrak{l}^{+}\right] } & =\left[\mathfrak{l}^{-}, \mathfrak{l}^{-}\right]=\emptyset
\end{aligned}
$$

where $\mathfrak{l}^{0}$ is $\mathfrak{p s u}(2 \mid 2) \oplus \mathfrak{p s u}(2 \mid 2) \oplus \mathfrak{u}(1) \oplus \mathbb{R}$ and is generated by $\left\{\mathrm{M}_{A B}, \mathrm{M}_{I J}, \mathrm{~S}_{a}, \overline{\mathrm{S}}_{a}, \mathrm{E}_{+}, \mathrm{E}_{-}\right\}$. We are using that $\mathfrak{s o}(4) \oplus \mathfrak{s o}(4) \simeq \mathfrak{s u}(2) \oplus \mathfrak{s u}(2) \oplus \mathfrak{s u}(2) \oplus \mathfrak{s u}(2)$. Before describing $\mathfrak{l}^{+}$and $\mathfrak{l}^{-}$we have to define the following combinations

$$
\begin{array}{ll}
\mathrm{A}_{A}=\mathrm{P}_{A}-\mathrm{i} \overline{\mathrm{P}}_{A}, \quad \mathrm{~A}_{A}^{\dagger}=\mathrm{P}_{A}+\mathrm{i} \overline{\mathrm{P}}_{A}, & \mathrm{~A}_{I}=\mathrm{P}_{I}-\mathrm{i} \overline{\mathrm{P}}_{I}, \quad \mathrm{~A}_{I}^{\dagger}=\mathrm{P}_{I}+\mathrm{i} \overline{\mathrm{P}}_{I}, \\
\mathrm{D}_{\dot{a}}=\frac{1}{\sqrt{2}}\left(\mathrm{~S}_{\dot{a}}-\mathrm{i} \Pi_{\dot{a} \dot{b}} \overline{\mathrm{S}}_{\dot{b}}\right), & \mathrm{D}_{\dot{a}}^{\dagger}=\frac{1}{\sqrt{2}}\left(\mathrm{~S}_{\dot{a}}+\mathrm{i} \Pi_{\dot{a} \dot{b}} \overline{\mathrm{S}}_{\dot{b}}\right)
\end{array}
$$

that satisfy a super Heisenberg algebra $\mathfrak{h}(8 \mid 8)$ with central element $\mathbb{E}_{-}$. The full Jordan decomposition is

$$
\begin{aligned}
\mathfrak{l}^{0} & =\mathfrak{p} \mathfrak{s u}(2 \mid 2) \oplus \mathfrak{p s u}(2 \mid 2) \oplus \mathfrak{u}(1) \oplus \mathbb{R}=\left\{\mathrm{M}_{A B}, \mathrm{M}_{I J}, \mathrm{~S}_{a}, \overline{\mathrm{S}}_{a}, \mathrm{E}_{+}, \mathrm{E}_{-}\right\} \\
\mathfrak{l}^{+} & =\left\{\mathrm{A}_{A}^{\dagger}, \mathrm{A}_{J}^{\dagger}, \mathrm{D}_{\dot{a}}^{\dagger}\right\}, \quad \mathfrak{l}^{-}=\left\{\mathrm{A}_{A}, \mathrm{~A}_{J}, \mathrm{D}_{\dot{a}}\right\} .
\end{aligned}
$$

It is straightforward to check that the relations (2.23) are satisfied.

Another interesting property of the contraction is that there exist a closed sub-algebra that has the same $\mathbb{Z}_{4}$ decomposition of the original $\mathfrak{p s u}(2,2 \mid 4)$. The decomposition is

$$
\mathfrak{g}_{0}=\left\{\overline{\mathrm{P}}_{A}, \overline{\mathrm{P}}_{I}\right\}, \quad \mathfrak{g}_{1}=\left\{\mathrm{S}_{\dot{a}}\right\}, \quad \mathfrak{g}_{2}=\left\{\mathrm{E}_{+}, \mathrm{E}_{-}, \mathrm{P}_{A}, \mathrm{P}_{I}\right\}, \quad \mathfrak{g}_{3}=\left\{\overline{\mathrm{S}}_{\dot{a}}\right\} .
$$

\footnotetext{
${ }^{1} \mathfrak{l}^{0}$ should not be confused with $\mathfrak{g}_{0}$ in the $\mathbb{Z}_{4}$ decomposition.
} 
The algebra generated by the operators above is $\mathfrak{h}(8 \mid 8) \rtimes \mathfrak{u}(1)$ where the $\mathfrak{u}(1)$ is generated by $\mathrm{E}_{+}$. Will see the coset $(H(8 \mid 8) \rtimes \mathrm{U}(1)) /\left(\mathbb{R}^{4} \times \mathbb{R}^{4}\right)$, where $\mathbb{R}^{4} \times \mathbb{R}^{4}$ is generated by $\left\{\overline{\mathrm{P}}_{A}, \overline{\mathrm{P}}_{I}\right\}$, plays an important role.

\subsection{Casimir of the contracted algebra and spectrum}

The quadratic Casimir for the $\mathfrak{p s u}(2,2 \mid 4)$ algebra written with the original generators is

$$
\begin{aligned}
\mathfrak{C}_{2}^{\mathfrak{p s u}}= & -\mathrm{T}^{2}+\delta^{A B} \mathrm{P}_{A} \mathrm{P}_{B}+J^{2}+\delta^{I J} \mathrm{P}_{I} \mathrm{P}_{J}-\frac{1}{R^{2}} \delta^{A B} \mathrm{M}_{A} \mathrm{M}_{B}-\frac{1}{R^{2}} \delta^{I J} \mathrm{M}_{I} \mathrm{M}_{J} \\
& -\frac{1}{2 R^{2}} \delta^{A C} \delta^{B D} \mathrm{M}_{A B} \mathrm{M}_{C D}+\frac{1}{2 R^{2}} \delta^{I K} \delta^{J L} \mathrm{M}_{I J} \mathrm{M}_{K L}-\frac{\mathrm{i}}{R} \Pi^{a b} \mathrm{Q}_{a} \overline{\mathrm{Q}}_{b}-\frac{\mathrm{i}}{R} \Pi^{\dot{a} \dot{b}} \mathrm{Q}_{\dot{a}} \overline{\mathrm{Q}}_{\dot{b}}
\end{aligned}
$$

In the limit $R \rightarrow \infty$ we get the quadratic Casimir of the $d=10$ super Poincare algebra $-\mathrm{T}^{2}+\delta^{A B} \mathrm{P}_{A} \mathrm{P}_{B}+\mathrm{J}^{2}+\delta^{I J} \mathrm{P}_{I} \mathrm{P}_{J}$. If we use the re-scalings defined before, we see that the surviving terms are

$$
\mathfrak{C}_{2}=-\mathrm{E}_{+} \mathrm{E}_{-}+\delta^{A B} \mathrm{P}_{A} \mathrm{P}_{B}+\delta^{I J} \mathrm{P}_{I} \mathrm{P}_{J}+\delta^{A B} \overline{\mathrm{P}}_{A} \overline{\mathrm{P}}_{B}+\delta^{I J} \overline{\mathrm{P}}_{I} \overline{\mathrm{P}}_{J}-i \Pi^{\dot{a} \dot{b}} \mathrm{~S}_{\dot{a}} \overline{\mathrm{S}}_{\dot{b}}
$$

One can check that is commutes with all generators. Note that the Casimir operator only contains generators of $\mathfrak{h}(8 \mid 8) \rtimes \mathfrak{u}(1)$. This indicates that all important physics happens in the smaller coset $(H(8 \mid 8) \rtimes \mathrm{U}(1)) /\left(\mathbb{R}^{4} \times \mathbb{R}^{4}\right)$. This is also related to the fact that the variables $\left(\theta^{a}, \bar{\theta}^{a}\right)$ that would be gauge fixed using kappa symmetry in the GS string.

Using the definitions of $\mathfrak{l}^{+}$and $\mathfrak{l}^{-}$above we can write the Casimir as

$$
\mathfrak{C}_{2}=-\mathrm{E}_{-} \mathrm{E}_{+}+\delta^{A B} \mathrm{~A}_{A}^{\dagger} \mathrm{A}_{B}+\delta^{I J} \mathrm{~A}_{I}^{\dagger} \mathrm{A}_{J}+\delta^{\dot{a} \dot{b}} \mathrm{D}_{\dot{a}}^{\dagger} \mathrm{D}_{\dot{b}},
$$

which has a more familiar form. We can build representations of the algebra starting with a vacuum $\left|E_{-}\right\rangle$satisfying [24]

$$
\mathrm{E}_{-}\left|E_{-}\right\rangle=E_{-}\left|E_{-}\right\rangle, \quad \mathrm{A}_{A}\left|E_{-}\right\rangle=\mathrm{A}_{I}\left|E_{-}\right\rangle=\mathrm{D}_{\dot{a}}\left|E_{-}\right\rangle=0 .
$$

Note that contrary to the flat space superstring the vacuum is not degenerate since $D_{\dot{a}}^{\dagger}$ changes the value of the energy measured in spacetime. The Casimir operator, which should correspond to the sum of the zero modes of the Virasoro operators $L_{0}+\bar{L}_{0}$ [19], kills $\left|E_{-}\right\rangle$if the eigenvalue of $\mathrm{E}_{+}$is equal to 0 . We can change this acting on $\left|E_{-}\right\rangle$with $\mathrm{D}_{\dot{a}}^{\dagger}$. This will change the eigenvalue of $\mathrm{E}^{-}$from 0 to 8 . The multiplet obtained in this way is the supergravity multiplet [17] which is composed of $(8+8) \times(8+8)=1+8+28+56+$ $70+56+28+8+1=256$ states. More explicitly, using that

$$
\left[\delta^{\dot{a} \dot{b}} \mathrm{D}_{\dot{a}}^{\dagger} \mathrm{D}_{\dot{b}}, \mathrm{D}_{\dot{c}}^{\dagger}\right]=-\mathrm{E}_{-} \mathrm{D}_{\dot{c}}^{\dagger}, \quad\left[\mathrm{E}_{+}, \mathrm{D}_{\dot{a}}^{\dagger}\right]=\mathrm{D}_{\dot{a}}^{\dagger}, \quad\left[\mathfrak{C}_{2}, \mathrm{D}_{\dot{a}}^{\dagger}\right]=0,
$$

the excited states are given by

$$
\left|E_{-}, \dot{a}\right\rangle=\mathrm{D}_{\dot{a}}^{\dagger}\left|E_{-}\right\rangle, \quad\left|E_{-}, \dot{a} \dot{b}\right\rangle=\mathrm{D}_{\dot{b}}^{\dagger} \mathrm{D}_{\dot{a}}^{\dagger}\left|E_{-}\right\rangle, \quad\left|E_{-}, \dot{a} \dot{b} \dot{c}\right\rangle=\mathrm{D}_{\dot{c}}^{\dagger} \mathrm{D}_{\dot{b}}^{\dagger} \mathrm{D}_{\dot{a}}^{\dagger}\left|E_{-}\right\rangle, \quad \text { etc. }
$$

and they all satisfy

$$
\mathfrak{C}_{2}|\Psi\rangle=0
$$

where $|\Psi\rangle$ is any state created acting with $\mathrm{D}_{\dot{a}}^{\dagger}$ (also with $\mathrm{A}_{A}^{\dagger}$ and $\mathrm{A}_{I}^{\dagger}$ ) on $\left|E_{-}\right\rangle$.

Later we will find an explicit description of these states in terms of unintegrated vertex operators. 


\section{$3 \quad$ Supergeometry}

The supergeometry is defined in terms of the coset element $g \in \frac{C P S U(2,2 \mid 4)}{\left(\mathbb{R}^{4} \rtimes \mathrm{SO}(4)\right) \times\left(\mathbb{R}^{4} \rtimes \mathrm{SO}(4)\right)}$. We will parametrize $g$ with the product of two factors. One depending only on $\mathrm{S}_{a}$ and $\overline{\mathrm{S}}_{a}$ and other with the remaining coset directions. The latter will be

$$
g\left(x^{+}, x^{-}, x^{A}, x^{I}, \theta^{\dot{a}}, \bar{\theta}^{\dot{a}}\right)=e^{\mathrm{i} x^{+} \mathrm{E}_{+}} e^{\mathrm{i}\left(x^{-} \mathrm{E}_{-}+x^{A} \mathrm{P}_{A}+x^{I} \mathrm{P}_{I}+\theta^{\dot{a}} \mathrm{~S}_{\dot{a}}+\bar{\theta}^{\dot{a}} \overline{\mathrm{S}}_{\dot{a}}\right) .}
$$

Note that the generators in the second factor above almost form an abelian algebra, the only non-trivial commutator is between the supercharges. Then we have that

$$
\begin{aligned}
& e^{-\mathrm{i}\left(x^{-} \mathrm{E}_{-}+x^{A} \mathrm{P}_{A}+x^{I} \mathrm{P}_{I}+\theta^{\dot{a}} \mathrm{~S}_{\dot{a}}+\bar{\theta}^{\dot{a}} \overline{\mathrm{S}}_{\dot{a}}\right)} d e^{\mathrm{i}\left(x^{-} \mathrm{E}_{-}+x^{A} \mathrm{P}_{A}+x^{I} \mathrm{P}_{I}+\theta^{\dot{a}} \mathrm{~S}_{\dot{a}}+\bar{\theta}^{\dot{a}} \overline{\mathrm{S}}_{\dot{a}}\right)}= \\
& \mathrm{i}\left(d x^{-}-\mathrm{i} d \theta^{\dot{a}} \theta^{\dot{a}}-\mathrm{i} d \bar{\theta}^{\dot{a}} \bar{\theta}^{\dot{a}}\right) \mathrm{E}_{-}+\mathrm{i} d x^{A} \mathrm{P}_{A}+\mathrm{i} d x^{I} \mathrm{P}_{I}+\mathrm{i} d \theta^{\dot{a}} \mathrm{~S}_{\dot{a}}+\mathrm{i} d \bar{\theta}^{\dot{a}} \overline{\mathrm{S}}_{\dot{a}} .
\end{aligned}
$$

The full current with $g\left(x^{+}, x^{-}, x^{A}, x^{I}, \theta^{\dot{a}}, \bar{\theta}^{\dot{a}}\right)$ will be

$$
\begin{aligned}
e^{-\mathrm{i}\left(x^{A} \mathrm{P}_{A}+x^{I} \mathrm{P}_{I}+\theta^{\dot{a}} \mathrm{~S}_{\dot{a}}+\bar{\theta}^{\dot{a}} \overline{\mathrm{S}}_{\dot{a}}\right)} \mathrm{i} d x^{+} \mathrm{E}_{+} e^{\mathrm{i}\left(+x^{A} \mathrm{P}_{A}+x^{I} \mathrm{P}_{I}+\theta^{\dot{a}} \mathrm{~S}_{\dot{a}}+\bar{\theta}^{\dot{a}} \overline{\mathrm{S}}_{\dot{a}}\right)} \\
+\mathrm{i}\left(d x^{-}-\mathrm{i} d \theta^{\dot{a}} \theta^{\dot{a}}-\mathrm{i} d \bar{\theta}^{\dot{a}} \bar{\theta}^{\dot{a}}\right) \mathrm{E}_{-}+\mathrm{i} d x^{A} \mathrm{P}_{A}+\mathrm{i} d x^{I} \mathrm{P}_{I}+\mathrm{i} d \theta^{\dot{a}} \mathrm{~S}_{\dot{a}}+\mathrm{i} d \bar{\theta}^{\dot{a}} \overline{\mathrm{S}}_{\dot{a}} .
\end{aligned}
$$

Because of the algebra, the expansion of the first term above stops in the terms quadratic in the fields

$$
\begin{aligned}
& J\left(x^{+}, x^{-}, x^{A}, x^{I}, \theta^{\dot{a}}, \bar{\theta}^{\dot{a}}\right)= \\
& \quad+\mathrm{i}\left(d x^{-}-\mathrm{i} d \theta^{\dot{a}} \theta^{\dot{a}}-\mathrm{i} d \bar{\theta}^{\dot{a}} \bar{\theta}^{\dot{a}}+d x^{+}\left(\frac{1}{4} x^{A} x^{A}+\frac{1}{4} x^{I} x^{I}+\mathrm{i} \theta_{\dot{a}} \bar{\theta}_{\dot{b}} \Pi_{\dot{a} \dot{b}}\right)\right) \mathrm{E}_{-} \\
& \quad+\mathrm{i} d x^{+} \mathrm{E}_{+}+\mathrm{i} d x^{A} \mathrm{P}_{A}+\mathrm{i} d x^{I} \mathrm{P}_{I}+\mathrm{i}\left(d \theta^{\dot{a}}+d x^{+} \bar{\theta}^{\dot{b}} \Pi_{\dot{b} \dot{a}}\right) \mathrm{S}_{\dot{a}}+\mathrm{i}\left(d \bar{\theta}^{\dot{a}}-d x^{+} \theta^{\dot{b}} \Pi_{\dot{b} \dot{a}}\right) \overline{\mathrm{S}}_{\dot{a}} \\
& \quad-\mathrm{i} d x^{+} x^{A} \overline{\mathrm{P}}_{A}-\mathrm{i} d x^{+} x^{I} \overline{\mathrm{P}}_{I} .
\end{aligned}
$$

The first terms are the vielbein and last two terms are the connections. This parametrization is the one that gives the usual metric in pp-wave backgrounds. This is not completely true because we still have to include $\theta^{a}$ and $\bar{\theta}^{a}$. Since the algebra with the corresponding generators only involves $\mathrm{E}_{-}, \mathrm{S}_{a}, \overline{\mathrm{S}}_{a}, \mathrm{M}_{A B}$ and $\mathrm{M}_{I J}$ we have that

$$
\begin{aligned}
& g^{-1}\left(\theta^{a}, \bar{\theta}^{a}\right) d g\left(\theta^{a}, \bar{\theta}^{a}\right)= \\
& \left(d \theta^{a} \theta^{a}+d \bar{\theta}^{a} \bar{\theta}^{a}\right) \mathrm{E}_{+}+\mathrm{i} E_{0}^{a}(\theta, \bar{\theta}) \mathrm{S}_{a}+\mathrm{i} \bar{E}_{0}^{a}(\theta, \bar{\theta}) \overline{\mathrm{S}}_{a}+\frac{1}{2} \Omega_{0}^{A B}(\theta, \bar{\theta}) M_{A B}+\frac{1}{2} \Omega_{0}^{I J}(\theta, \bar{\theta}) \mathrm{M}_{I J},
\end{aligned}
$$

where the differential forms $\left(E^{a}, \bar{E}^{a}, \Omega^{A B}, \Omega^{I J}\right)$ are constrained by the Maurer-Cartan identity $d J+J \wedge J=0$. Since this sub-algebra is symmetric under the switch $\mathrm{S}_{a} \leftrightarrow \overline{\mathrm{S}}_{a}$ we have that $\bar{E}_{0}^{a}(\theta, \bar{\theta})=E_{0}^{a}(\bar{\theta}, \theta)$ and $\Omega_{0}(\theta, \bar{\theta})=\Omega_{0}(\bar{\theta}, \theta)$. The Maurer-Cartan identities in components are

$$
\begin{array}{r}
d \theta^{a} \wedge d \theta^{a}+d \bar{\theta}^{a} \wedge d \bar{\theta}^{a}+E_{0}^{a} \wedge E_{0}^{a}+\bar{E}_{0}^{a} \wedge \bar{E}_{0}^{a}=0, \\
d E_{0}^{a}+E_{0}^{b} \wedge \Omega_{0}^{A B} \sigma_{A B}^{b a}+E_{0}^{b} \wedge \Omega_{0}^{I J} \sigma_{I J}^{b a}=0, \\
d \bar{E}_{0}^{a}+\bar{E}_{0}^{b} \wedge \Omega_{0}^{A B} \sigma_{A B}^{b a}+\bar{E}_{0}^{b} \wedge \Omega_{0}^{I J} \sigma_{I J}^{b a}=0, \\
d \Omega_{0}^{A B}+\Omega_{0}^{C D} \wedge \Omega_{0}^{E F} f_{C D}^{A B} E F=0, \\
d \Omega_{0}^{I J}+\Omega_{0}^{K L} \wedge \Omega_{0}^{M N} f_{K L M N}^{I J}=0,
\end{array}
$$


where $f_{C D E F}^{A B}$ and $f_{K L M N}^{I J}$ are $\mathfrak{s o}(4)$ structure constants. The explicit expression of these forms can be found [16], however we hopefully will not need them.

The have to full geometry we multiply the two coset factors as

$$
g=g\left(\theta^{a}, \bar{\theta}^{a}\right) g\left(x^{+}, x^{-}, x^{A}, x^{I}, \theta^{\dot{a}}, \bar{\theta}^{\dot{a}}\right),
$$

and the full Maurer-Cartan current is

$$
\begin{aligned}
g^{-1} d g= & J\left(x^{+}, x^{-}, x^{A}, x^{I}, \theta^{\dot{a}}, \bar{\theta}^{\dot{a}}\right) \\
& +e^{-\mathrm{i}\left(x^{A} \mathrm{P}_{A}+x^{I} \mathrm{P}_{I}+\theta^{\dot{a}} \mathrm{~S}_{\dot{a}}+\bar{\theta}^{\dot{a}} \overline{\mathrm{S}}_{\dot{a}}\right)} g^{-1}\left(\theta^{a}, \bar{\theta}^{a}\right) d g\left(\theta^{a}, \bar{\theta}^{a}\right) e^{\mathrm{i}\left(x^{A} \mathrm{P}_{A}+x^{I} \mathrm{P}_{I}+\theta^{\dot{a}} \mathrm{~S}_{\dot{a}}+\bar{\theta}^{\dot{a}} \overline{\mathrm{S}}_{\dot{a}}\right),}
\end{aligned}
$$

the expansion of the second term stops at quadratic order in $\left(x^{A}, x^{I}, \theta^{\dot{a}}, \bar{\theta}^{\dot{a}}\right)$.

The expansion is

$$
\begin{aligned}
g^{-1} d g= & \mathrm{i} J^{+} \mathrm{E}_{+}+\mathrm{i} J^{-} \mathrm{E}_{-}+\mathrm{i} J^{A} \mathrm{P}_{A}+\mathrm{i} J^{I} \mathrm{P}_{I}+\mathrm{i} J_{1}^{a} \mathrm{~S}_{a}+\mathrm{i} J_{1}^{\dot{a}} \mathrm{~S}_{\dot{a}} \\
& +\mathrm{i} J_{3}^{a} \overline{\mathrm{S}}_{a}+\mathrm{i} J_{3}^{\dot{a}} \overline{\mathrm{S}}_{\dot{a}}+\frac{1}{2} \Omega^{A B} \mathrm{M}_{A B}+\frac{1}{2} \Omega^{I J} \mathrm{M}_{I J}+i \Omega^{A} \overline{\mathrm{P}}_{A}+i \Omega^{I} \overline{\mathrm{P}}_{I}
\end{aligned}
$$

where

$$
\begin{aligned}
J^{+}= & d x^{+}-\mathrm{i}\left(d \theta_{a} \theta_{a}+d \bar{\theta}_{a} \bar{\theta}_{a}\right), \\
J^{-}= & d x^{-}-\mathrm{i}\left(d \theta_{\dot{a}} \theta_{\dot{a}}+d \bar{\theta}_{\dot{a}} \bar{\theta}_{\dot{a}}\right) \\
& +\left(d x^{+}-\mathrm{i}\left(d \theta_{a} \theta_{a}+d \bar{\theta}_{a} \bar{\theta}_{a}\right)\right)\left(\frac{1}{4} x^{A} x^{A}+\frac{1}{4} x^{I} x^{I}+\mathrm{i} \theta_{\dot{c}} \bar{\theta}_{\dot{d}} \Pi_{\dot{c} \dot{d}}\right) \\
& +\frac{\mathrm{i}}{2}\left(\left(E_{0} X \Pi \bar{\theta}\right)-\left(\bar{E}_{0} X \Pi \theta\right)\right)-\frac{\mathrm{i}}{8}((\theta \dot{\Omega} \theta)+(\bar{\theta} \dot{\Omega} \bar{\theta})), \\
J^{A}= & d x^{A}-\mathrm{i}\left(E_{0} \sigma^{A} \theta+\bar{E}_{0} \sigma^{A} \bar{\theta}\right)-\Omega_{0}^{A B} X^{B}, \\
J^{I}= & d x^{I}-\mathrm{i}\left(E_{0} \sigma^{I} \theta+\bar{E}_{0} \sigma^{I} \bar{\theta}\right)-\Omega_{0}^{I J} X^{J}, \\
J_{1}^{\dot{a}}= & d \theta^{\dot{a}}-\frac{1}{4}(\dot{\Omega} \theta)^{\dot{a}}+\left(d x^{+}-\mathrm{i}\left(d \theta^{a} \theta^{a}+d \bar{\theta}^{a} \bar{\theta}^{a}\right)\right)(\Pi \bar{\theta})^{\dot{a}}+\frac{1}{2}\left(\bar{E}_{0} X \Pi\right)^{\dot{a}}, \\
J_{3}^{\dot{a}}= & d \bar{\theta}^{\dot{a}}-\frac{1}{4}(\dot{\Omega} \bar{\theta})^{\dot{a}}-\left(d x^{+}-\mathrm{i}\left(d \theta^{a} \theta^{a}+d \bar{\theta}^{a} \bar{\theta}^{a}\right)\right)(\Pi \theta)^{\dot{a}}-\frac{1}{2}\left(E_{0} X \Pi\right)^{\dot{a}}, \\
\Omega^{A}= & -x^{A}\left(d x^{+}-\mathrm{i}\left(d \theta^{a} \theta^{a}+d \bar{\theta}^{a} \bar{\theta}^{a}\right)\right)-\mathrm{i}\left(E_{0} \sigma^{A} \Pi \bar{\theta}-\bar{E}_{0} \sigma^{A} \Pi \theta\right), \\
\Omega^{I}= & -x^{I}\left(d x^{+}-\mathrm{i}\left(d \theta^{a} \theta^{a}+d \bar{\theta}^{a} \bar{\theta}^{a}\right)\right)-\mathrm{i}\left(E_{0} \sigma^{I} \Pi \bar{\theta}-\bar{E}_{0} \sigma^{I} \Pi \theta\right), \\
J_{1}^{a}= & E_{0}^{a}, \quad J_{3}^{a}=\bar{E}_{0}^{a}, \quad \Omega^{A B}=\Omega_{0}^{A B}, \quad \Omega^{I J}=\Omega_{0}^{I J},
\end{aligned}
$$

where we are using the following notations to have more compact expressions

$$
\begin{aligned}
X_{a \dot{a}} & =x_{A} \sigma_{a \dot{a}}^{A}+x_{I} \sigma_{a \dot{a}}^{I}, & \dot{\Omega}_{\dot{a} \dot{b}} & =\Omega_{0}^{A B}\left(\sigma_{A B}\right)_{\dot{a} \dot{b}}+\Omega_{0}^{I J}\left(\sigma_{I J}\right)_{\dot{a} \dot{b}}, \\
E_{0}^{a}\left(\sigma_{A}\right)_{a \dot{a}} \theta^{\dot{a}} & =\left(E_{0} \sigma_{A} \theta\right), & E_{0}^{a}\left(\sigma_{A} \Pi\right)_{a \dot{a}} \bar{\theta}^{\dot{a}} & =\left(E_{0} \sigma_{A} \Pi \bar{\theta}\right), \\
\bar{E}_{0}^{a}\left(\sigma_{A}\right)_{a \dot{a}} \bar{\theta}^{\dot{a}} & =\left(\bar{E}_{0} \sigma_{A} \bar{\theta}\right), & \bar{E}_{0}^{a}\left(\sigma_{A} \Pi\right)_{a \dot{a}} \theta^{\dot{a}} & =\left(\bar{E}_{0} \sigma_{A} \Pi \theta\right),
\end{aligned}
$$

and similar expressions.

The currents above define the whole supergeometry of the plane wave background. They define the frame fields $d Z^{M} E_{M} \bullet$, where $\bullet$ is any of the $\mathfrak{g}_{1}, \mathfrak{g}_{2}$ or $\mathfrak{g}_{3}$ directions and 
connections $d Z^{M} \Omega_{M}{ }^{\circ}$ where $\circ$ is any of the $\mathfrak{g}_{0}$ directions. The index $M$ is a local coordinate index for the coset element $g\left(Z^{M}\right)$. In our explicit parametrization this is identified with the $\bullet$ indices . The covariant derivatives are defined with the inverse of $E_{M} \bullet$ and $\Omega_{M}{ }^{\circ}$

$$
\nabla_{\bullet}=E_{\bullet}{ }^{M}\left(\partial_{M}-\Omega_{M}{ }^{\circ} M_{\circ}\right) .
$$

For now we will restrict to the supergeometry of the coset $(H(8 \mid 8) \rtimes \mathrm{U}(1)) /\left(\mathbb{R}^{4} \times \mathbb{R}^{4}\right)$. From (3.4) we can read off $E_{M} \bullet$ and $\Omega_{M}{ }^{\circ}$ for this case and write the covariant derivatives after inverting the vielbein

$$
\begin{array}{ll}
\nabla_{-}=\partial_{-}, & \nabla_{+}=\partial_{+}-\frac{1}{4} x^{2} \partial_{-}-\bar{\theta}_{\dot{a}} \Pi_{\dot{a} \dot{b}} \partial_{\dot{b}}+\theta_{\dot{a}} \Pi_{\dot{a} \dot{b}} \bar{\partial}_{\dot{b}}+\mathrm{i} x^{A} \overline{\mathrm{P}}_{A}+\mathrm{i} x^{I} \overline{\mathrm{P}}_{I}, \\
\nabla_{A}=\partial_{A}, & \nabla_{I}=\partial_{I}, \quad \nabla_{\dot{a}}=\partial_{\dot{a}}+\frac{\mathrm{i}}{2} \theta_{\dot{a}} \partial_{-}, \quad \bar{\nabla}_{\dot{a}}=\bar{\partial}_{\dot{a}}+\frac{\mathrm{i}}{2} \bar{\theta}_{\dot{a}} \partial_{-}, \\
\bar{\nabla}_{A}=\overline{\mathrm{P}}_{A}, & \bar{\nabla}_{I}=\overline{\mathrm{P}}_{I},
\end{array}
$$

where $\left(\bar{\nabla}_{A}, \bar{\nabla}_{I}\right)$ are the generators of the boosts in the directions $A$ and $I$. The $\left(\overline{\mathrm{P}}_{A}, \overline{\mathrm{P}}_{I}\right)$ should be understood as acting on coset elements $g$ by multiplication from the right. Therefore they are defined to satisfy

$$
\begin{array}{ll}
{\left[\nabla_{+}, \overline{\mathrm{P}}_{A}\right]=\mathrm{i} \nabla_{A},} & {\left[\nabla_{+}, \overline{\mathrm{P}}_{I}\right]=-\mathrm{i} \nabla_{I},} \\
{\left[\nabla_{A}, \overline{\mathrm{P}}_{B}\right]=\frac{\mathrm{i}}{2} \delta_{A B} \nabla_{-},} & {\left[\nabla_{I}, \overline{\mathrm{P}}_{J}\right]=-\frac{\mathrm{i}}{2} \delta_{I J} \nabla_{-} .}
\end{array}
$$

Furthermore, the isotropy generators do not act on the coordinates. This is because there is no linear variation of coordinates such that $g^{-1} \delta g=v^{A} \overline{\mathrm{P}}_{A}+v^{I} \overline{\mathrm{P}}_{I}$. It can be verified that the algebra of these covariant derivatives is the same as their corresponding generators but with an extra -i multiplying the structure constants. This is because, by definition, the covariant derivatives are differential operators such that when acting in the coset element $g$ we have that

$$
\nabla \bullet g=\mathrm{i} g \boldsymbol{\top}_{\bullet},
$$

where $T_{\bullet}$ is the corresponding algebra generator. Similarly, the isometry generators can also be represented as differential operators with the property that

$$
\mathrm{t}_{\bullet} g=\mathrm{T}_{\bullet} g \text {. }
$$

The reason for the different hermiticity conventions is that usually one wants covariant derivatives that are anti-hermitian, but symmetry generators are usually hermitian. The expressions for the symmetry generators are

$$
\begin{aligned}
& \mathrm{e}_{-}=-\mathrm{i} \partial_{-}=, \quad \mathrm{e}_{+}=-\mathrm{i} \partial_{+}, \\
& \mathrm{p}_{A}=-\mathrm{i} \cos \left(x^{+}\right) \partial_{A}+\sin \left(x^{+}\right) \overline{\mathrm{P}}_{A}+\frac{\mathrm{i}}{2} \sin \left(x^{+}\right) x_{A} \partial_{-}, \\
& \overline{\mathrm{p}}_{A}=\cos \left(x^{+}\right) \overline{\mathrm{P}}_{A}+\mathrm{i} \sin \left(x^{+}\right) \partial_{A}+\frac{\mathrm{i}}{2} \cos \left(x^{+}\right) x_{A} \partial_{-}
\end{aligned}
$$




$$
\begin{aligned}
& \mathrm{p}_{I}=-\mathrm{i} \cos \left(x^{+}\right) \partial_{I}+\sin \left(x^{+}\right) \overline{\mathrm{P}}_{I}+\frac{\mathrm{i}}{2} \sin \left(x^{+}\right) x_{I} \partial_{-}, \\
& \overline{\mathrm{p}}_{I}=\cos \left(x^{+}\right) \overline{\mathrm{P}}_{I}+\mathrm{i} \sin \left(x^{+}\right) \partial_{I}+\frac{\mathrm{i}}{2} \cos \left(x^{+}\right) x_{I} \partial_{-} \\
& \mathrm{s}_{\dot{a}}=-\mathrm{i} \cos \left(x^{+}\right) \mathrm{q}_{\dot{a}}-\mathrm{i} \sin \left(x^{+}\right) \Pi_{\dot{a} \dot{b}} \overline{\mathrm{q}}_{\dot{b}}, \\
& \overline{\mathrm{s}}_{\dot{a}}=-\mathrm{i} \cos \left(x^{+}\right) \overline{\mathrm{q}}_{\dot{a}}+w \mathrm{i} \sin \left(x^{+}\right) \Pi_{\dot{a} \dot{b}} \mathrm{q}_{\dot{b}},
\end{aligned}
$$

where

$$
\mathrm{q}_{\dot{a}}=\partial_{\dot{a}}-\frac{\mathrm{i}}{2} \theta_{\dot{a}} \partial_{-}, \quad \overline{\mathrm{q}}_{\dot{a}}=\bar{\partial}_{\dot{a}}-\frac{\mathrm{i}}{2} \bar{\theta}_{\dot{a}} \partial_{-} .
$$

The operators $\left(\overline{\mathrm{P}}_{A}, \overline{\mathrm{P}}_{I}\right)$ above are the same as the ones used in the covariant derivative, so it should be understood as acting on $g$ from the right. This also means they commute with all the partial derivatives above. Using this we can check that

$$
\left[\mathrm{e}_{+}, \mathrm{p}_{A}\right]=-\mathrm{i} \overline{\mathrm{p}}_{A}, \quad\left[\mathrm{p}_{A}, \overline{\mathrm{p}}_{B}\right]=\frac{\mathrm{i}}{2} \mathrm{e}_{-}, \quad\left[\mathrm{e}_{+}, \overline{\mathrm{p}}_{A}\right]=\mathrm{ip}_{A}, \quad \text { etc. }
$$

The origin of the additional minus sign is from the definition (3.33)

$$
\mathrm{t}_{1} \mathrm{t}_{2} g=\mathrm{t}_{1} \mathrm{~T}_{2} g=\mathrm{T}_{2} \mathrm{t}_{1} g=\mathrm{T}_{2} \mathrm{~T}_{1} g .
$$

If we include dependence on the remaining odd directions there will be further contributions to all operators above. In particular we note that $\left(\overline{\mathrm{p}}_{A}, \overline{\mathrm{p}}_{I}\right)$ will get contributions like

$$
\begin{aligned}
\overline{\mathrm{p}}_{A}\left(\theta^{a}, \bar{\theta}^{a}\right) & =\overline{\mathrm{p}}_{A}^{(0)}-\frac{1}{2}\left(\theta \sigma_{A}\right)_{\dot{a}} \mathrm{~s}_{\dot{a}}^{(0)}-\frac{1}{2}\left(\bar{\theta} \sigma_{A}\right)_{\dot{a}} \overline{\mathrm{s}}_{\dot{a}}^{(0)}+\cdots, \\
\overline{\mathrm{p}}_{I}\left(\theta^{a}, \bar{\theta}^{a}\right) & =\overline{\mathrm{p}}_{I}^{(0)}-\frac{1}{2}\left(\theta \sigma_{I}\right)_{\dot{a}} \mathrm{~s}_{\dot{a}}^{(0)}-\frac{1}{2}\left(\bar{\theta} \sigma_{I}\right)_{\dot{a}} \overline{\mathrm{s}}_{\dot{a}}^{(0)}+\cdots \\
\mathrm{p}_{A}\left(\theta^{a}, \bar{\theta}^{a}\right) & =\mathrm{p}_{A}^{(0)}+\frac{1}{2}\left(\theta \sigma_{A} \Pi\right)_{\dot{a}} \overline{\mathrm{s}}_{\dot{a}}^{(0)}-\frac{1}{2}\left(\bar{\theta} \sigma_{A} \Pi\right)_{\dot{a}} \dot{s}_{\dot{a}}^{(0)}+\cdots \\
\mathrm{p}_{I}\left(\theta^{a}, \bar{\theta}^{a}\right) & =\mathrm{p}_{I}^{(0)}+\frac{1}{2}\left(\theta \sigma_{I} \Pi\right)_{\dot{a}} \overline{\mathrm{s}}_{\dot{a}}^{(0)}-\frac{1}{2}\left(\bar{\theta} \sigma_{I} \Pi\right)_{\dot{a}} \mathrm{~s}_{\dot{a}}^{(0)}+\cdots
\end{aligned}
$$

Similarly, the operators $\left(\mathrm{s}_{\dot{a}}, \overline{\mathrm{s}}_{\dot{a}}\right)$ get the contributions

$$
\begin{aligned}
& \mathrm{s}_{\dot{a}}\left(\theta^{a}, \bar{\theta}^{a}\right)=\mathrm{s}_{\dot{a}}^{(0)}-\mathrm{i}\left(\theta \sigma_{i}\right)_{\dot{a}} \mathrm{p}_{i}^{(0)}+\mathrm{i}\left(\bar{\theta} \sigma_{i} \Pi\right)_{\dot{a}} \overline{\mathbf{p}}_{i}^{(0)}+\cdots \\
& \overline{\mathbf{s}}_{\dot{a}}\left(\theta^{a}, \bar{\theta}^{a}\right)=\overline{\mathrm{s}}_{\dot{a}}^{(0)}-\mathrm{i}\left(\bar{\theta} \sigma_{i}\right)_{\dot{a}} \mathrm{p}_{i}^{(0)}-\mathrm{i}\left(\theta \sigma_{i} \Pi\right)_{\dot{a}} \overline{\mathbf{p}}_{i}^{(0)}+\cdots
\end{aligned}
$$

We will use these expressions later. It will also be important that the expression for the differential generator $\mathbf{e}_{+}$does not change in the full coset

$$
\mathrm{e}_{+}=\mathrm{e}_{+}^{(0)}=-\mathrm{i} \partial_{+}
$$

\section{Action, BRST and conformal invariance}

In this section we will review the sigma model action for the pure spinor string in the plane wave background [18] and prove its invariance under the BRST-like transformations. As 
in the $A d S_{5} \times S^{5}$ case, the geometric part of the action is constructed using the MaurerCartan one-form $J=g^{-1} d g$, where $g$ is a coset element. This one-form is expanded in the algebra elements as

$$
\begin{aligned}
J= & \mathrm{i} J^{+} \mathrm{E}_{+}+\mathrm{i} J^{-} \mathrm{E}_{-}+\mathrm{i} J^{A} \mathrm{P}_{A}+\mathrm{i} J^{I} \mathrm{P}_{I}+\mathrm{i} J_{1}^{a} \mathrm{~S}_{a}+\mathrm{i} J_{1}^{\dot{a}} \mathrm{~S}_{\dot{a}} \\
& +\mathrm{i} J_{3}^{a} \overline{\mathrm{S}}_{a}+\mathrm{i} J_{3}^{\dot{a}} \overline{\mathrm{S}}_{\dot{a}}+\frac{1}{2} \Omega^{A B} \mathrm{M}_{A B}+\frac{1}{2} \Omega^{I J} \mathrm{M}_{I J}+i \Omega^{A} \overline{\mathrm{P}}_{A}+i \Omega^{I} \overline{\mathrm{P}}_{I} .
\end{aligned}
$$

The world-sheet action is

$$
\begin{aligned}
S=\int d^{2} z & \left(-J^{+} \bar{J}^{-}-J^{-} \bar{J}^{+}+\frac{1}{2} J^{A} \bar{J}^{A}+\frac{1}{2} J^{I} \bar{J}^{I}+d_{a} \bar{J}_{1}^{a}+d_{\dot{a}} \bar{J}_{1}^{\dot{a}}+\bar{d}_{a} J_{3}^{a}+\bar{d}_{\dot{a}} J_{3}^{\dot{a}}\right. \\
& \left.+\frac{\mathrm{i}}{2} \Pi^{\dot{a} \dot{b}} d_{\dot{a}} \bar{d}_{\dot{b}}+\omega_{a} \bar{\nabla} \lambda^{a}+\omega_{\dot{a}} \bar{\nabla} \lambda^{\dot{a}}+\bar{\omega}_{a} \nabla \bar{\lambda}^{a}+\bar{\omega}_{\dot{a}} \nabla \bar{\lambda}^{\dot{a}}-N_{A} \bar{N}_{A}-N_{I} \bar{N}_{I}\right)+S_{\mathrm{WZ}} .
\end{aligned}
$$

This action uses the following definitions. The fields $\left(\lambda^{a}, \lambda^{\dot{a}}, \bar{\lambda}^{a}, \bar{\lambda}^{\dot{a}}\right)$ are the pure spinor ghosts that satisfy

$$
\lambda^{a} \sigma_{a \dot{a}}^{A} \lambda^{\dot{a}}=\lambda^{a} \sigma_{a \dot{a}}^{I} \lambda^{\dot{a}}=\bar{\lambda}^{a} \sigma_{a \dot{a}}^{A} \bar{\lambda}^{\dot{a}}=\bar{\lambda}^{a} \sigma_{a \dot{a}}^{I} \bar{\lambda}^{\dot{a}}=\lambda^{a} \lambda^{a}=\lambda^{\dot{a}} \lambda^{\dot{a}}=\bar{\lambda}^{a} \bar{\lambda}^{a}=\bar{\lambda}^{\dot{a}} \bar{\lambda}^{\dot{a}}=0
$$

The fields $\left(\omega_{a}, \omega_{\dot{a}}, \bar{\omega}_{a}, \bar{\omega}_{\dot{a}}\right)$ are their conjugate momenta. The covariant derivatives are

$$
\begin{array}{rlrl}
\bar{\nabla} \lambda^{a} & =\bar{\partial} \lambda^{a}-\frac{1}{2} \bar{\Omega}^{a b} \lambda^{b}, & \nabla \bar{\lambda}^{a}=\partial \bar{\lambda}^{a}-\frac{1}{2} \Omega^{a b} \bar{\lambda}^{b}, \\
\bar{\nabla} \lambda^{\dot{a}}=\bar{\partial} \lambda^{\dot{a}}-\frac{1}{2} \bar{\Omega}^{\dot{a} \dot{b}} \lambda^{\dot{b}}-\frac{1}{2} \bar{\Omega}^{\dot{a} a} \lambda^{a}, & \nabla \bar{\lambda}^{\dot{a}}=\partial \bar{\lambda}^{\dot{a}}-\frac{1}{2} \Omega^{\dot{a} \dot{b}} \bar{\lambda}^{\dot{b}}-\frac{1}{2} \Omega^{\dot{a} a} \bar{\lambda}^{a},
\end{array}
$$

where

$$
\begin{aligned}
& \Omega^{a b}=\frac{1}{2} \Omega^{A B}\left(\sigma_{A B}\right)^{a b}+\frac{1}{2} \Omega^{I J}\left(\sigma_{I J}\right)^{a b}, \quad \Omega^{\dot{a} \dot{b}}=\frac{1}{2} \Omega^{A B}\left(\sigma_{A B}\right)^{\dot{a} \dot{b}}+\frac{1}{2} \Omega^{I J}\left(\sigma_{I J}\right)^{\dot{a} \dot{b}}, \\
& \Omega^{\dot{a} a}=\Omega^{A}\left(\sigma_{A}\right)^{a \dot{a}}+\Omega^{I}\left(\sigma_{I}\right)^{a \dot{a}},
\end{aligned}
$$

and analogous expressions for the left-moving connections. The reason for the asymmetry in the definitions of covariant derivatives is that after the contraction, only the spinor with dot type index transforms under boosts in the $A$ and $I$ directions. The new currents in the second line are

$$
N_{A}=\frac{1}{2} \lambda_{a} \omega_{\dot{b}}\left(\sigma_{A}\right)_{a \dot{b}}, \quad N_{I}=\frac{1}{2} \lambda_{a} \omega_{\dot{b}}\left(\sigma_{I}\right)_{a \dot{b}}, \quad \bar{N}_{A}=\frac{1}{2} \bar{\lambda}_{a} \bar{\omega}_{\dot{a}}\left(\sigma_{A}\right)_{a \dot{a}}, \quad \bar{N}_{I}=\frac{1}{2} \bar{\lambda}_{a} \bar{\omega}_{\dot{a}}\left(\sigma_{I}\right)_{a \dot{a}} .
$$

We can also define

$$
\begin{aligned}
N^{A B} & =\frac{1}{2} \lambda_{a} \omega_{b}\left(\sigma^{A B}\right)_{a b}+\frac{1}{2} \lambda_{\dot{a}} \omega_{\dot{b}}\left(\sigma^{A B}\right)_{\dot{a} \dot{b}}, & N^{I J} & =\frac{1}{2} \lambda_{a} \omega_{b}\left(\sigma^{I J}\right)_{a b}+\frac{1}{2} \lambda_{\dot{a}} \omega_{\dot{b}}\left(\sigma^{I J}\right)_{\dot{a} \dot{b}}, \\
\bar{N}^{A B} & =\frac{1}{2} \bar{\lambda}_{a} \bar{\omega}_{b}\left(\sigma^{A B}\right)_{a b}+\frac{1}{2} \bar{\lambda}_{\dot{a}} \bar{\omega}_{\dot{b}}\left(\sigma^{A B}\right)_{\dot{a} \dot{b}}, & \bar{N}^{I J} & =\frac{1}{2} \bar{\lambda}_{a} \bar{\omega}_{b}\left(\sigma^{I J}\right)_{a b}+\frac{1}{2} \bar{\lambda}_{\dot{a}} \bar{\omega}_{\dot{b}}\left(\sigma^{I J}\right)_{\dot{a} \dot{b}} .
\end{aligned}
$$

Note that using all these definitions, the kinetic terms for the ghosts can be written as

$$
\begin{aligned}
& \omega_{a} \bar{\nabla} \lambda^{a}+\omega_{\dot{a}} \bar{\nabla} \lambda^{\dot{a}}+\bar{\omega}_{a} \nabla \bar{\lambda}^{a}+\bar{\omega}_{\dot{a}} \nabla \bar{\lambda}^{\dot{a}}=\omega_{a} \bar{\partial} \lambda^{a}+\omega_{\dot{a}} \bar{\partial} \lambda^{\dot{a}}+\bar{\omega}_{a} \partial \bar{\lambda}^{a}+\bar{\omega}_{\dot{a}} \partial \bar{\lambda}^{\dot{a}} \\
& -\frac{1}{2} \bar{\Omega}^{A B} N_{A B}-\frac{1}{2} \Omega^{A B} \bar{N}_{A B}-\frac{1}{2} \Omega^{I J} \bar{N}_{I J}-\frac{1}{2} \bar{\Omega}^{I J} N_{I J}-\bar{\Omega}^{A} N_{A}-\bar{\Omega}^{I} N_{I}-\Omega^{A} \bar{N}_{A}-\Omega^{I} \bar{N}_{I} .
\end{aligned}
$$


Finally, $S_{\mathrm{WZ}}$ is the Wess-Zumino term which is defined on a three-dimensional surface whose boundary is world-sheet of the string [25] and its most compact form is

$$
S_{\mathrm{WZ}}=k \int_{\Sigma_{3}} \gamma_{m \alpha \beta}\left(J_{2}^{m} \wedge J_{1}^{\alpha} \wedge J_{1}^{\beta}-J_{2}^{m} \wedge J_{3}^{\alpha} \wedge J_{3}^{\beta}\right)
$$

where $\gamma_{m \alpha \beta}$ is the $\gamma$-matrix in ten dimensional notation. Unlike the $A d S_{5} \times S^{5}$ case, the WZ term cannot be written as an integral of a globally defined two-form for the plane wave background. Despite this fact, nevertheless, as usual expected for a Wess-Zumino term, any variation of $S_{W Z}$ can be written as an integral at the boundary. If a variation along the coset directions is parametrized by $\epsilon=g^{-1} \delta g$, (4.12) transforms to

$$
\int\left(\epsilon_{2}^{m}\left(J_{1}^{\alpha} \wedge J_{1}^{\beta}-J_{3}^{\alpha} \wedge J_{3}^{\beta}\right)+\epsilon_{1}^{\alpha}\left(J_{1}^{\beta} \wedge J_{2}^{m}+J_{2}^{m} \wedge J_{1}^{\alpha}\right)-\epsilon_{3}^{\alpha}\left(J_{3}^{\beta} \wedge J_{2}^{m}+J_{2}^{m} \wedge J_{3}^{\beta}\right)\right) \gamma_{m \alpha \beta} .
$$

For the geometric part of the action, the BRST-like transformation for the coset element $g$ is $Q g=g(\lambda+\bar{\lambda})$ where $\lambda=\lambda^{a} \mathrm{~S}_{a}+\lambda^{\dot{a}} \mathrm{~S}_{\dot{a}}$ and $\bar{\lambda}=\bar{\lambda}^{a} \overline{\mathrm{S}}_{a}+\bar{\lambda}^{\dot{a}} \overline{\mathrm{S}}_{\dot{a}}$. The transformation of $J=g^{-1} d g$ is $Q J=d(\lambda+\bar{\lambda})+[J, \lambda+\bar{\lambda}]$. Using the algebra from section 2 we obtain

$$
\begin{aligned}
Q J^{+} & =\lambda^{a} J_{1}^{a}+\bar{\lambda}^{a} J_{3}^{a}, \quad Q J^{-}=\lambda^{\dot{a}} J_{1}^{\dot{a}}+\bar{\lambda}^{\dot{a}} J_{3}^{\dot{a}}, \\
Q J_{2}^{A} & =\left(\lambda \sigma^{A} J_{1}\right)+\left(J_{1} \sigma^{A} \lambda\right)+\left(\bar{\lambda} \sigma^{A} J_{3}\right)+\left(J_{3} \sigma^{A} \bar{\lambda}\right), \\
Q J_{2}^{I} & =\left(\lambda \sigma^{I} J_{1}\right)+\left(J_{1} \sigma^{I} \lambda\right)+\left(\bar{\lambda} \sigma^{I} J_{3}\right)+\left(J_{3} \sigma^{I} \bar{\lambda}\right) \\
Q J_{1}^{a} & =-\mathrm{i} \nabla \lambda^{a}, \quad Q J_{1}^{\dot{a}}=-\mathrm{i} \nabla \lambda^{\dot{a}}-\mathrm{i} J_{2}^{+}(\bar{\lambda} \Pi)^{\dot{a}}+\frac{\mathrm{i}}{2} J_{2}^{A}\left(\bar{\lambda} \sigma_{A} \Pi\right)^{\dot{a}}+\frac{\mathrm{i}}{2} J_{2}^{I}\left(\bar{\lambda} \sigma_{I} \Pi\right)^{\dot{a}}, \\
Q J_{3}^{a} & =-\mathrm{i} \nabla \bar{\lambda}^{a}, \quad Q J_{3}^{\dot{a}}=-\mathrm{i} \nabla \bar{\lambda}^{\dot{a}}+\mathrm{i} J_{2}^{+}(\lambda \Pi)^{\dot{a}}-\frac{\mathrm{i}}{2} J_{2}^{A}\left(\lambda \sigma_{A} \Pi\right)^{\dot{a}}-\frac{\mathrm{i}}{2} J_{2}^{I}\left(\lambda \sigma_{I} \Pi\right)^{\dot{a}} .
\end{aligned}
$$

From the same calculation we also obtain the BRST-like transformations of the connections

$$
\begin{aligned}
Q \Omega^{A B} & =\left(\lambda^{a} J_{3}^{b}+\bar{\lambda}^{b} J_{1}^{a}\right)\left(\sigma^{A B} \Pi\right)_{a b} \\
Q \Omega^{I J} & =-\left(\lambda^{a} J_{3}^{b}+\bar{\lambda}^{b} J_{1}^{a}\right)\left(\sigma^{I J} \Pi\right)_{a b} \\
Q \Omega^{A} & =\left(\lambda^{a} J_{3}^{\dot{b}}+\bar{\lambda}^{\dot{b}} J_{1}^{a}\right)\left(\sigma^{A} \Pi\right)_{a \dot{b}}+\left(\lambda^{\dot{a}} J_{3}^{b}+\bar{\lambda}^{b} J_{1}^{\dot{a}}\right)\left(\sigma^{A} \Pi\right)_{\dot{a} b} \\
Q \Omega^{I} & =\left(\lambda^{a} J_{3}^{\dot{b}}+\bar{\lambda}^{\dot{b}} J_{1}^{a}\right)\left(\sigma^{I} \Pi\right)_{a \dot{b}}-\left(\lambda^{\dot{a}} J_{3}^{b}+\bar{\lambda}^{b} J_{1}^{\dot{a}}\right)\left(\sigma^{I} \Pi\right)_{\dot{a} b} .
\end{aligned}
$$

It remains to define the transformations of the fields not defined by the geometry. The pure spinor ghost variables $\lambda, \bar{\lambda}$ are BRST invariant and the pure spinor antighosts transform as

$$
Q \omega_{a}=-\mathrm{i} d_{a}, \quad Q \omega_{\dot{a}}=-\mathrm{i} d_{\dot{a}}, \quad Q \bar{\omega}_{a}=-\mathrm{i} \bar{d}_{a}, \quad Q \bar{\omega}_{\dot{a}}=-\mathrm{i} \bar{d}_{\dot{a}} .
$$

The last fields are the supersymmetric momenta

$$
\begin{aligned}
Q d_{a}= & 2 \lambda_{a} J_{2}^{-}-J^{A}\left(\sigma_{A} \lambda\right)_{a}-J^{I}\left(\sigma_{I} \lambda\right)_{a}+\frac{1}{2}\left(N^{A B}\left(\sigma_{A B} \Pi \bar{\lambda}\right)_{a}-N^{I J}\left(\sigma_{I J} \Pi \bar{\lambda}\right)_{a}\right) \\
& +\left(N_{A}\left(\sigma_{A} \Pi \bar{\lambda}\right)_{a}+N_{I}\left(\sigma_{I} \Pi \bar{\lambda}\right)_{a}\right) \\
Q d_{\dot{a}}= & 2 \lambda^{\dot{a}} J^{+}-J_{2}^{A}\left(\lambda \sigma_{A}\right)_{\dot{a}}-J_{2}^{I}\left(\lambda \sigma_{I}\right)_{\dot{a}}+\left(N_{A}\left(\sigma_{A} \Pi \bar{\lambda}\right)_{\dot{a}}-N_{I}\left(\sigma_{I} \Pi \bar{\lambda}\right)_{\dot{a}}\right)
\end{aligned}
$$




$$
\begin{aligned}
Q \bar{d}_{a}= & 2 \bar{\lambda}_{a} \bar{J}_{2}^{-}-\bar{J}_{2}^{A}\left(\sigma_{A} \bar{\lambda}\right)_{a}-\bar{J}_{2}^{I}\left(\sigma_{I} \bar{\lambda}\right)_{a}+\frac{1}{2}\left(\bar{N}^{A B}\left(\lambda \sigma_{A B} \Pi\right)_{a}-\bar{N}^{I J}\left(\lambda \sigma_{I J} \Pi\right)_{a}\right) \\
& +\left(\bar{N}_{A}\left(\lambda \sigma_{A} \Pi\right)_{a}-\bar{N}_{I}\left(\lambda \sigma_{I} \Pi\right)_{a}\right) \\
Q \bar{d}_{\dot{a}}= & 2 \bar{\lambda}_{\dot{a}} \bar{J}_{2}^{+}-\bar{J}_{2}^{A}\left(\bar{\lambda} \sigma_{A}\right)_{\dot{a}}-\bar{J}_{2}^{I}\left(\bar{\lambda} \sigma_{I}\right)_{\dot{a}}+\left(\bar{N}_{A}\left(\lambda \sigma_{A} \Pi\right)_{\dot{a}}-\bar{N}_{I}\left(\lambda \sigma_{I} \Pi\right)_{\dot{a}}\right) .
\end{aligned}
$$

The calculation of the BRST transformation of the action can be organized as follows. We first note that for the case of a BRST transformation the $\epsilon$ in (4.13) is given by $\lambda^{a} S_{a}+\lambda^{\dot{a}} S_{\dot{a}}+\bar{\lambda}^{a} \bar{S}_{a}+\bar{\lambda}^{\dot{a}} \bar{S}_{\dot{a}}$. In this case, it simplifies to

$$
\begin{aligned}
& Q S_{W Z}=k \int d^{2} z( \lambda^{a} \\
&\left(J^{[-} \bar{J}^{a]}-\frac{1}{2} J^{[i} \bar{J}^{\dot{b}]} \sigma_{a \dot{a}}^{i}\right)+\lambda^{\dot{a}}\left(J^{[+} \bar{J}^{\dot{a}]}-\frac{1}{2} J^{[i} \bar{J}^{b]} \sigma_{b \dot{a}}^{i}\right) \\
&\left.-\bar{\lambda}^{a}\left(J^{[-} \bar{J}^{a]}-\frac{1}{2} J^{[i} \bar{J}^{\dot{b}]} \sigma_{\alpha \dot{b}}^{i}\right)-\bar{\lambda}^{\dot{a}}\left(J^{[+} \bar{J}^{\dot{a}]}-\frac{1}{2} J^{[i} \bar{J}^{b]} \sigma_{b \dot{a}}^{i}\right)\right) .
\end{aligned}
$$

The strange anti-symmetrization in different type of indices should actually be read off as an anti-symmetrization of the left- and right-moving currents. It turns out that if the constant $k$ is equal to 1 the BRST transformation of $\int d^{2} z\left(-J^{(+} \bar{J}^{-)}+\frac{1}{2} J^{i} \bar{J}^{i}\right)+S_{W Z}$ is

$$
\begin{aligned}
\int d^{2} z( & \left(-2 \lambda^{a} J_{2}^{-}+\lambda^{\dot{b}} J_{2}^{A} \sigma_{a \dot{b}}^{A}+\lambda^{\dot{b}} J^{I} \sigma_{a \dot{b}}^{I}\right) \bar{J}_{1}^{a}+\left(-2 \lambda^{\dot{a}} J^{+}+\lambda^{b} J_{2}^{A} \sigma_{b \dot{a}}^{A}+\lambda^{b} J_{2}^{I} \sigma_{b \dot{a}}^{I}\right) \bar{J}_{1}^{\dot{a}} \\
& \left.+\left(-2 \bar{\lambda}^{a} \bar{J}^{-}+\bar{\lambda}^{\dot{b}} \bar{J}_{2}^{A} \sigma_{a \dot{b}}^{A}+\bar{\lambda}^{b} \bar{J}_{2}^{I} \sigma_{a \dot{b}}^{I}\right) J_{3}^{a}+\left(-2 \bar{\lambda}^{\dot{a}} \bar{J}_{2}^{+}+\bar{\lambda}^{b} \bar{J}_{2}^{A} \sigma_{b \dot{a}}^{A}+\bar{\lambda}^{b} \bar{J}_{2}^{I} \sigma_{b \dot{a}}^{I}\right) J_{3}^{\dot{a}}\right)
\end{aligned}
$$

This expression is canceled by some terms in BRST transformations of fields $\left(d_{a}, d_{\dot{a}}, \bar{d}_{a}, \bar{d}_{\dot{a}}\right)$ in $\int\left(d_{a} \bar{J}_{1}^{a}+d_{\dot{a}} \bar{J}_{1}^{\dot{a}}+\bar{d}_{a} J_{3}^{a}+\bar{d}_{\dot{a}} J_{3}^{\dot{a}}+\frac{i}{2} \Pi^{\dot{a} \dot{b}} d_{\dot{a}} \bar{d}_{\dot{b}}\right)$. The terms from $Q d$ that remain, together with the transformations of $\left(\bar{J}_{1}^{a}, \bar{J}_{1}^{\dot{a}}, J_{3}^{a}, J_{3}^{\dot{a}}\right)$ will cancel with the transformations of the anti-ghosts and connections in the ghost part of the action.

It was argued in [18] that the action (4.2) is conformally invariant to all orders in perturbation theory. The argument goes as follows. Using the supergeometry defined in section 3 we can calculate the explicit form of the action in terms of the parametrization for $g$. If we assign a positive charge to $\left(\theta^{a}, \bar{\theta}^{a}\right)$ and a negative charge to $\left(d_{a}, \bar{d}_{a}\right)$ we can separate the action in two parts. One part has positive charge and the other has zero charge. We will call this $S$-charge. This comes from the expansion of the Maurer-Cartan currents $\left(J^{\bullet}, J^{\circ}\right)$. The part that has zero $S$-charge contains the kinetic term for $\left(\theta^{a}, d_{a}, \bar{\theta}^{a}, \bar{d}_{a}\right)$, the ghosts and a coset sigma model generated by $\left\{\mathrm{E}_{+}, \mathrm{E}_{-}, \mathrm{P}_{A}, \mathrm{P}_{I}, \mathrm{~S}_{\dot{a}}, \overline{\mathrm{S}}_{\dot{a}}, \overline{\mathrm{P}}_{A}, \overline{\mathrm{P}}_{I}\right\} /\left\{\overline{\mathrm{P}}_{A}, \overline{\mathrm{P}}_{I}\right\}$. Because of the structure of the vertices in the coset sigma model and ghosts, the divergent part of the effective action of the zero $S$-charge part has only a one loop contribution, which vanishes. Since the zero $R$-charge part is tied to the positive charge part by the isometry transformations and that the propagator for $\left(\theta^{a}, d_{a}, \bar{\theta}^{a}, \bar{d}_{a}\right)$ conserves the $S$-charge, it follows that the whole action is conformally invariant to all loop orders. Only the full Maurer-Cartan current $\left(J^{\bullet}, J^{\circ}\right)$ is invariant under all isometries. This drastic simplification comes from the fact there is no Ramond-Ramond flux coupling the fields $\left(d_{a}, \bar{d}_{a}\right)$ with the rest of the variables. In the following section we will argue that, at least for the massless sector, the physical spectrum can be found by looking at unintegrated vertex operators with zero $S$-charge. 


\section{Massless vertex operators}

Vertex operators in string theory comes in two flavors, unintegrated and integrated. They describe the same spectrum and both are need to compute observables. In the pure spinor formalism they are related by a chain of equations [26] that follows from the BRST-like symmetry of the theory. The integrated vertex are interpreted as deformations of the action.

The unintegrated vertex operators are space-time scalars with conformal vanishing world-sheet conformal dimension. For massless states this means they are constructed with world-sheet scalars and its anomalous dimension has to vanish. Their general form is $U\left(\lambda^{a}, \bar{\lambda}^{a}, \lambda^{\dot{a}}, \bar{\lambda}^{\dot{a}}, g\left(Z^{M}\right)\right)$. The cohomology defined by the BRST-like transformations implies that at ghost number zero the only physical operator is the identity. At ghost number one, the cohomology is found to be related to the conserved currents corresponding to the space-time global symmetries [27]. The massless spectrum is in the ghost number two cohomology. Using the $\mathfrak{s o}(4) \oplus \mathfrak{s o}(4)$ notation we write

$$
\begin{aligned}
U\left(\lambda^{a}, \bar{\lambda}^{a}, \lambda^{\dot{a}}, \bar{\lambda}^{\dot{a}}, g\left(Z^{M}\right)\right)= & \lambda^{a} \bar{\lambda}^{b} U_{a b}\left(g\left(Z^{M}\right)\right)+\lambda^{a} \bar{\lambda}^{\dot{b}} U_{a \dot{b}}\left(g\left(Z^{M}\right)\right) \\
& +\lambda^{\dot{a}} \bar{\lambda}^{b} U_{\dot{a} b}\left(g\left(Z^{M}\right)\right)+\lambda^{\dot{a}} \bar{\lambda}^{\dot{b}} U_{\dot{a} \dot{b}}\left(g\left(Z^{M}\right)\right) .
\end{aligned}
$$

The physical state conditions comes from the condition that $U\left(\lambda^{a}, \bar{\lambda}^{a}, \lambda^{\dot{a}}, \bar{\lambda}^{\dot{a}}, g\left(Z^{M}\right)\right)$ is invariant under the BRST-like transformations. Since the ghosts are invariant, the only contribution comes from the coset element $\delta g=g\left(\lambda^{a} \mathrm{~S}_{a}+\lambda^{\dot{a}} \mathrm{~S}_{\dot{a}}+\bar{\lambda}^{a} \overline{\mathrm{S}}_{a}+\bar{\lambda}^{\dot{a}} \overline{\mathrm{S}}_{\dot{a}}\right)$. For a general function of $g$, we have that

$$
f(g+\delta g)=f(g)+\left(\lambda^{a} \nabla_{a}+\lambda^{\dot{a}} \nabla_{\dot{a}}+\bar{\lambda}^{a} \bar{\nabla}_{a}+\bar{\lambda}^{\dot{a}} \bar{\nabla}_{\dot{a}}\right) f(g),
$$

where $\left(\nabla_{a}, \nabla_{\dot{a}}, \bar{\nabla}_{a}, \bar{\nabla}_{\dot{a}}\right)$ are the covariant derivatives defined in section 3 .

As in the case of the action, we can expand a general function $f(g)$ of the coset in $S$-charge powers

$$
f(g)=\sum_{n=0}^{16} f^{(n)}(g) .
$$

The term with zero $S$-charge is a function of only the smaller coset $(H(8 \mid 8) \rtimes \mathrm{U}(1)) /\left(\mathbb{R}^{4} \times\right.$ $\mathbb{R}^{4}$ ). We will denote an element of the this coset by $g_{0}$. So we have that

$$
f(g)=f\left(g_{0}\right)+\sum_{n=1}^{16} f^{(n)}(g) .
$$

If the function is a space-time scalar it must be invariant under all isometries, in particular it must be invariant under the isometries generated by $\left(\overline{\mathrm{p}}_{A}, \overline{\mathrm{p}}_{I}\right)$

$$
f\left(g^{\prime}\right)=f(\Lambda g)=f(g),
$$

where $\Lambda$ is a finite isometry transformation. From the algebra we can see that, for example, the isometry transformations along the directions $\left\{\overline{\mathrm{p}}_{A}, \overline{\mathrm{p}}_{I}\right\}$ raise the $S$-charge. It follows from (3.44) that

$$
\delta_{A} \theta^{a}=0, \quad \delta_{A} \theta^{\dot{a}}=\frac{\dot{\mathrm{i}}}{2}\left(\theta \sigma_{A}\right)^{\dot{a}}
$$


The consequence of this is that all different $S$-charge powers of $f(g)$ are tied together by global isometry invariance. This is very similar to the argument used to prove conformal invariance of the full action once the vanishing $S$-charge part was found to be finite [18]. Therefore we will first find what is appropriate vertex operator starting with functions of the smaller coset $f\left(g_{0}\right)$.

Imposing that $U$ must be invariant under all isometries is too strong. For example, in flat space, if we demand that a vertex operator is invariant under all translations its momentum should vanish. For the coset, the only possible invariant combinations that are invariant under all isometries are the differentials $g^{-1} d g$ and finite differences $g_{1}^{-1} g_{2}$. Later in this section we will discuss what are the appropriate conditions to impose on $U$ such that we find its full superspace form.

Suppose we can find a single vertex operator $U\left(x^{-}, x^{+}, x^{A}, x^{I}, \theta^{\dot{a}}, \bar{\theta}^{\dot{a}}, \lambda, \bar{\lambda}\right)$ corresponding to a scalar that satisfy $Q U=0$ and that depends only on a scalar polarization that are invariant under some of the isometries. If such operator exists we can construct the full $U$ order by order in $\left(\theta^{a}, \bar{\theta}^{a}\right)$. It is crucial that the generators $\left(\overline{\mathrm{P}}_{A}, \overline{\mathrm{P}}_{I}\right)$ inside the differential form of the isometry generators act only on the ghosts and polarizations.

We will now construct a scalar vertex operator. First we start with an ansatz that depends only on $\left(x^{-}, \theta^{\dot{a}}, \bar{\theta}^{\dot{a}}\right)$. The general form of the vertex operator will be

$$
\begin{aligned}
U_{0}\left(x^{-}, \theta^{\dot{a}}, \bar{\theta}^{\dot{a}}, \lambda, \bar{\lambda}\right)= & \lambda^{a} \bar{\lambda}^{b} U_{a b}\left(x^{-}, \theta^{\dot{a}}, \bar{\theta}^{\dot{a}}\right)+\lambda^{\dot{a}} \bar{\lambda}^{b} U_{\dot{a} b}\left(x^{-}, \theta^{\dot{a}}, \bar{\theta}^{\dot{a}}\right) \\
& +\lambda^{a} \bar{\lambda}^{\dot{b}} U_{a \dot{b}}\left(x^{-}, \theta^{\dot{a}}, \bar{\theta}^{\dot{a}}\right)+\lambda^{\dot{a}} \bar{\lambda}^{\dot{b}} U_{\dot{a} \dot{b}}\left(x^{-}, \theta^{\dot{a}}, \bar{\theta}^{\dot{a}}\right) .
\end{aligned}
$$

Two of the equations that come from BRST invariance are

$$
\nabla_{\dot{a}} U_{\dot{b} a}+\nabla_{\dot{b}} U_{\dot{a} a}=\delta_{\dot{a} \dot{b}} A_{a}, \quad \bar{\nabla}_{\dot{a}} U_{a \dot{b}}+\bar{\nabla}_{\dot{b}} U_{a \dot{a}}=\delta_{\dot{a} \dot{b}} \bar{A}_{a} .
$$

If $\nabla_{-} U\left(x^{-}, \theta^{\dot{a}}, \bar{\theta}^{\dot{a}}, \lambda, \bar{\lambda}\right) \neq 0$, the case where this vanishes will be seen later, these equations can be solved as

$$
U_{\dot{a} a}=\frac{\nabla_{\dot{a}}}{2 \nabla_{-}} A_{a}, \quad U_{a \dot{a}}=\frac{\bar{\nabla}_{\dot{a}}}{2 \nabla_{-}} \bar{A}_{a},
$$

but these can be canceled by a gauge transformation for the vertex. Since the component $U_{\dot{a} \dot{b}}$ is related with the ones above by the $\left(\overline{\mathrm{P}}_{A}, \overline{\mathrm{P}}_{I}\right)$ isotropies, it will also vanish. Only $\left(\lambda^{a}, \bar{\lambda}^{a}\right)$ are invariant under these isotropies. The remaining equations are

$$
\nabla_{\dot{a}} U_{a b}=\left(\sigma^{A}\right)_{a \dot{a}} U_{b}^{A}+\left(\sigma^{I}\right)_{a \dot{a}} U_{b}^{I}, \quad \bar{\nabla}_{\dot{a}} U_{a b}=\left(\sigma^{A}\right)_{b \dot{a}} \bar{U}_{a}^{A}+\left(\sigma^{I}\right)_{b \dot{a}} \bar{U}_{a}^{I} .
$$

These equations can be solved with two known superspace functions $f_{a}\left(E_{-}, \theta^{\dot{a}}, \eta^{A}, \eta^{I}, \eta_{a}\right)$ and $\bar{f}_{a}\left(E_{-}, \bar{\theta}^{\dot{a}}, \bar{\eta}^{A}, \bar{\eta}^{I}, \bar{\eta}_{a}\right)$ that satisfy

$$
\left(\partial_{\dot{a}}-\frac{E_{-}}{2} \theta_{\dot{a}}\right) f_{a}=\left(\sigma^{A}\right)_{a \dot{a}} f^{A}+\left(\sigma^{I}\right)_{a \dot{a}} f^{I}, \quad\left(\bar{\partial}_{\dot{a}}-\frac{E_{-}}{2} \bar{\theta}_{\dot{a}}\right) \bar{f}_{a}=\left(\sigma^{A}\right)_{a \dot{a}} f^{A}+\left(\sigma^{I}\right)_{a \dot{a}} \bar{f}^{I},
$$

that depend on the set of polarizations $\left(\eta^{A}, \eta^{I}, \eta_{a}, \bar{\eta}^{A}, \bar{\eta}^{I}, \bar{\eta}_{a}\right)$. Their explicit expressions can be found in $[20,28,29]$. Up to now our ansatz for the unintegrated vertex operator is

$$
U_{0}\left(x^{-}, \theta^{\dot{a}}, \bar{\theta}^{\dot{a}}, \lambda^{a}, \bar{\lambda}^{a}\right)=\lambda^{a} \bar{\lambda}^{b} f_{a}\left(E_{-}, \theta^{\dot{a}}, \eta^{A}, \eta^{I}, \eta_{a}\right) \bar{f}_{a}\left(E_{-}, \bar{\theta}^{\dot{a}}, \bar{\eta}^{A}, \bar{\eta}^{I}, \bar{\eta}_{a}\right) e^{\mathrm{i} E_{-} x^{-}} .
$$


It should be noted that $x^{-}$is not a periodic variable so $E_{-}$can have any real value. The functions $\left(f_{a}, \bar{f}_{a}\right)$ are singular in the limit $E_{-} \rightarrow 0$. However, in the plane wave background we can construct a scalar superfield that is well defined in this limit

$$
\Phi\left(E_{-}, \eta^{A}, \eta^{I}, \eta^{a}, \bar{\eta}^{A}, \bar{\eta}^{I}, \bar{\eta}^{a}\right)=\left(E_{-}\right)^{2} \Pi^{a b} f_{a}\left(E_{-}, \theta^{\dot{a}}, \eta^{A}, \eta^{I}, \eta_{a}\right) \bar{f}_{a}\left(E_{-}, \bar{\theta}^{\dot{a}}, \bar{\eta}^{A}, \bar{\eta}^{I}, \bar{\eta}_{a}\right) .
$$

In the limit $E_{-} \rightarrow 0$ the superfield reduces to

$$
\Phi\left(0, \eta^{A}, \eta^{I}, \eta^{a}, \bar{\eta}^{A}, \bar{\eta}^{I}, \bar{\eta}^{a}\right)=4 \Pi^{a b} \eta_{a} \bar{\eta}_{b} .
$$

The scalar $\Phi$ is the field that changes the background value of the Ramond-Ramond flux by a constant amount $[30,31]$. However for $E_{-} \neq 0$ it is not a scalar since it depends on the polarizations inside the functions $\left(f_{a}, \bar{f}_{a}\right)$. In order to have a scalar we will set

$$
\begin{aligned}
\eta^{A} & =\eta^{I}=\bar{\eta}^{A}=\bar{\eta}^{I}=0, \\
\eta_{a} \bar{\eta}_{b} & =\left(E_{-}\right)^{2} \Pi_{a b} .
\end{aligned}
$$

This initial ansatz for the unintegrated vertex operator is then

$$
U_{0}\left(x^{-}, \theta^{\dot{a}}, \bar{\theta}^{\dot{a}}, \lambda^{a}, \bar{\lambda}^{a}\right)=\left.\lambda^{a} \bar{\lambda}^{a} f_{a}\left(E_{-}, \theta^{\dot{a}}, 0,0, \eta_{a}\right) \bar{f}_{a}\left(E_{-}, \bar{\theta}^{\dot{a}}, 0,0, \bar{\eta}_{a}\right) e^{\mathrm{i} E_{-} x^{-}}\right|_{\eta_{a} \bar{\eta}_{b}=\left(E_{-}\right)^{2} \Pi_{a b}} .
$$

In the expression above we have set $\phi=1$. We will include it explicitly when discussing the construction of the massless spectrum.

The next step is to introduce dependence on the remaining bosonic coordinates. Since we are working with the smaller coset we are missing the constraints imposed by $\lambda^{a} \nabla_{a}+$ $\bar{\lambda}^{a} \bar{\nabla}_{a}$. In particular, the full Virasoro constraint cannot be obtained if this part of the BRST transformation is not included. We will take another route and impose that the vertex operator is killed by the Casimir operator $\mathfrak{C}_{2}(2.30)$ in differential form. The consequence is that one effectively imposes $L_{0}+\bar{L}_{0}=0$ on the state. This can be done using the covariant derivatives or the symmetry generators. The quadratic Casimir evaluated with both set of operators differ by a minus sign due to the different hermiticity convention

$$
\mathfrak{C}_{2}=-\partial_{+} \partial_{-}+\frac{x^{2}}{4} \partial_{-}^{2}+\partial_{A} \partial_{A}+\partial_{I} \partial_{I}-\mathrm{i} \Pi^{\dot{a} \dot{b}} \mathbf{s}_{\dot{a}} \bar{s}_{\dot{b}}
$$

Since the vertex operators are invariant under isotropy transformations we drop $\left(\overline{\mathrm{P}}_{A}, \overline{\mathrm{P}}_{I}\right)$. Notice that the quadratic Casimir can be written as

$$
\begin{aligned}
\mathfrak{C}_{2}= & -\partial_{-}\left(\partial_{+}+4 \mathrm{i}\right)+\left(\partial_{A}+\mathrm{i} \frac{x_{A}}{2} \partial_{-}\right)\left(\partial_{A}-\mathrm{i} \frac{x_{A}}{2} \partial_{-}\right) \\
& +\left(\partial_{I}+\mathrm{i} \frac{x_{I}}{2} \partial_{-}\right)\left(\partial_{I}-\mathrm{i} \frac{x_{I}}{2} \partial_{-}\right)-\mathrm{i} \Pi^{\dot{a} \dot{b}} \mathbf{s}_{\dot{a}} \overline{\mathrm{s}}_{\dot{b}} .
\end{aligned}
$$

We will be able make the identification

$$
U\left(x^{-}, x^{+}, x^{A}, x^{I}, \theta^{\dot{a}}, \bar{\theta}^{\dot{a}}, \lambda^{a}, \bar{\lambda}^{a}\right) \leftrightarrow\left|E_{-}\right\rangle,
$$


if $U$ satisfy the vacuum conditions described in section 2.2. However, in order to have a normalizable state, the choice of creation and annihilation operators depend on the sign of $E_{-}$. The complex linear combinations inside $\mathfrak{C}_{2}$ are precisely the ones that appear in

$$
\begin{array}{rlrl}
\mathrm{p}_{A}+\mathrm{i} \overline{\mathrm{p}}_{A} & =e^{-\mathrm{i} x^{+}}\left(-\mathrm{i} \partial_{A}-\mathrm{i} \frac{1}{2} x_{A} E_{-}\right), & \mathrm{p}_{A}-\mathrm{i} \overline{\mathrm{p}}_{A}=e^{\mathrm{i} x^{+}}\left(-\mathrm{i} \partial_{A}+\mathrm{i} \frac{1}{2} x_{A} E_{-}\right) \\
\mathrm{p}_{I}+\mathrm{i} \overline{\mathrm{p}}_{I}=e^{-\mathrm{i} x^{+}}\left(-\mathrm{i} \partial_{I}-\mathrm{i} \frac{1}{2} x_{I} E_{-}\right), & \mathrm{p}_{I}-\mathrm{i} \overline{\mathrm{p}}_{I}=e^{\mathrm{i} x^{+}}\left(-\mathrm{i} \partial_{I}+\mathrm{i} \frac{1}{2} x_{I} E_{-}\right),
\end{array}
$$

where we are dropping dependence on $\left(\overline{\mathrm{P}}_{A}, \overline{\mathrm{P}}_{I}\right)$ and, when acting on $U_{0}, \partial_{-}$can be identified with $\mathrm{i} E_{-}$. The operators above exactly like the creation-annihilation operators of harmonic oscillators. So we add the dependence on the bosonic coordinates as the wave function of the ground state of an harmonic oscillator. This is not surprising since in the light-cone GS description of the superstring the bosonic directions are massive world-sheet fields.

From now on we will assume $E_{-}$is positive. This means $U$ will be normalizable if it is annihilated by

$$
\left(\mathrm{p}_{A}+\mathrm{i} \overline{\mathrm{p}}_{A}\right) U=\left(\mathrm{p}_{I}+\mathrm{i}_{I}\right) U=0 .
$$

Up to now the unintegrated vertex operator is

$$
U\left(x^{-}, x^{A}, x^{I}, \theta^{\dot{a}}, \bar{\theta}^{\dot{a}}, \lambda^{a}, \bar{\lambda}^{a}\right)=e^{-\frac{E_{-}}{4} x^{2}} U_{0}\left(x^{-}, \theta^{\dot{a}}, \bar{\theta}^{\dot{a}}, \lambda^{a}, \bar{\lambda}^{a}\right),
$$

where $x^{2}=x^{A} x^{A}+x^{I} x^{I}$.

To get the final constraint on superspace and fix the $x^{+}$dependence we need to define the Clifford vacuum, as discussed in detail in [17]. First we write the quadratic Casimir as

$$
\begin{aligned}
\mathfrak{c}_{2}= & \partial_{-} \partial_{+}+\left(\partial_{A}+\mathrm{i} \frac{x_{A}}{2} \partial_{-}\right)\left(\partial_{A}-\mathrm{i} \frac{x_{A}}{2} \partial_{-}\right) \\
& +\left(\partial_{I}+\mathrm{i} \frac{x_{I}}{2} \partial_{-}\right)\left(\partial_{I}-\mathrm{i} \frac{x_{I}}{2} \partial_{-}\right)+\frac{1}{2} \delta_{\dot{a} \dot{c}}\left(\mathbf{s}_{\dot{a}}+\mathrm{i} \Pi_{\dot{a} \dot{b}} \overline{\mathbf{s}}_{\dot{b}}\right)\left(\mathrm{s}_{\dot{c}}-\mathrm{i} \Pi_{\dot{c} \dot{d}} \overline{\mathbf{s}}_{\dot{d}}\right) .
\end{aligned}
$$

If we choose the vertex operator $U$ to be annihilated by $s_{\dot{a}}-i \Pi_{\dot{a} \dot{b}} \overline{\mathrm{s}}_{\dot{b}}$ its $\mathrm{e}_{+}$eigenvalue will be 0 . This choice is the same from the one made in section 2.2. We can study this condition using a chiral basis. First we define

$$
\kappa^{\dot{a}}=\frac{1}{2}\left(\theta^{\dot{a}}+\mathrm{i} \Pi^{\dot{a} \dot{b}} \bar{\theta}^{\dot{b}}\right), \quad \bar{\kappa}^{\dot{a}}=\frac{1}{2}\left(\theta^{\dot{a}}-\mathrm{i} \Pi^{\dot{a} \dot{b}} \bar{\theta}^{\dot{b}}\right) .
$$

Next we define a chiral variable

$$
\tilde{x}^{-}=x^{-}+\mathrm{i} \kappa^{\dot{a}} \bar{\kappa}^{\dot{a}} .
$$

The complex combinations of the zero and one $S$-charge supersymmetry generators $\left(\mathrm{s}_{\dot{a}}, \overline{\mathrm{s}}_{\dot{a}}\right)$ also have simple expressions in terms of $\left(\tilde{x}^{-}, \kappa^{\dot{a}}, \bar{\kappa}^{\dot{a}}\right)$

$$
\begin{aligned}
& \mathrm{s}_{\dot{a}}+\mathrm{i} \Pi_{\dot{a} \dot{b}} \overline{\mathrm{s}}_{\dot{b}}=-\mathrm{i} e^{-\mathrm{i} x^{+}}\left(\frac{\partial}{\partial \bar{\kappa}^{\dot{a}}}-2 \mathrm{i} \kappa_{\dot{a}} \tilde{\partial}_{-}\right)-\mathrm{i}\left(\theta \sigma^{i}+\mathrm{i} \bar{\theta} \sigma_{i} \Pi\right)_{\dot{a}}\left(\mathrm{p}_{i}^{(0)}+\mathrm{i} \bar{p}_{i}^{(0)}\right)+\cdots, \\
& \mathrm{s}_{\dot{a}}-\mathrm{i} \Pi_{\dot{a} \dot{b}} \overline{\mathrm{s}}_{\dot{b}}=-\mathrm{i} e^{\mathrm{i} x^{+}} \frac{\partial}{\partial \kappa^{\dot{a}}}-\mathrm{i}\left(\theta \sigma^{i}-\mathrm{i} \bar{\theta} \sigma_{i} \Pi\right)_{\dot{a}}\left(\mathrm{p}_{i}^{(0)}-\mathrm{i} \bar{p}_{i}^{(0)}\right)+\cdots
\end{aligned}
$$


The condition on $U$ can be written in terms of supersymmetry generators as

$$
\left(\mathrm{s}_{\dot{a}}-\mathrm{i} \Pi_{\dot{a} \dot{b}} \overline{\mathrm{s}}_{\dot{a}}\right) U_{\text {final }}\left(\tilde{x}^{-}, x^{+}, x^{A}, x^{I}, \theta^{\dot{a}}, \bar{\theta}^{\dot{a}}, \theta^{a}, \bar{\theta}^{a}, \lambda^{a}, \bar{\lambda}^{a}\right)=0 .
$$

If we restrict to the zero $S$-charge part of $\mathrm{s}_{\dot{a}}-i \Pi_{\dot{a} \dot{b}} \overline{\mathrm{s}}_{\dot{b}}$ there would be no solution to this condition that is compatible with BRST invariance. However, if we include the higher $\left(\theta^{a}, \bar{\theta}^{a}\right)$ contributions in (3.48) and (3.49) we can solve iteratively in powers of $\left(\theta^{a}, \bar{\theta}^{a}\right)$.

Using all the expressions above, we can see that the vertex operator satisfying the BPS condition (5.32) is

$$
\begin{aligned}
& U_{\text {final }}\left(x^{-}, x^{A}, x^{I}, \theta^{\dot{a}}, \bar{\theta}^{\dot{a}}, \theta^{a}, \bar{\theta}^{a}, \lambda^{a}, \bar{\lambda}^{a}\right)= \\
& \quad U^{(0)}\left(x^{-}, x^{A}, x^{I}, \theta^{\dot{a}}, \bar{\theta}^{\dot{a}}, \lambda^{a}, \bar{\lambda}^{a}\right)+\theta^{a} U_{a}^{(1)}+\bar{\theta}^{a} \bar{U}_{a}^{(1)}+\cdots,
\end{aligned}
$$

where

$$
\begin{aligned}
& U^{(0)}\left(x^{-}, x^{A}, x^{I}, \theta^{\dot{a}}, \bar{\theta}^{\dot{a}}, \lambda^{a}, \bar{\lambda}^{a}\right)= \\
& \left.\quad \lambda^{a} \bar{\lambda}^{a} f_{a}\left(E_{-}, \theta^{\dot{a}}, 0,0, \eta_{a}\right) \bar{f}_{a}\left(E_{-}, \bar{\theta}^{\dot{a}}, 0,0, \bar{\eta}_{a}\right) e^{\mathrm{i} E_{-} \tilde{x}^{-}-\frac{E_{-}}{4} x^{2}}\right|_{\eta_{a} \bar{\eta}_{b}=\left(E_{-}\right)^{2} \Pi_{a b}}
\end{aligned}
$$

and $\left(U_{a}^{(1)}, \bar{U}_{a}^{(1)}\right)$ satisfy

$$
\begin{aligned}
\frac{\partial}{\partial \kappa^{\dot{a}}} U_{a}^{(1)} & =-\mathrm{i} E_{-} \sigma_{a \dot{a}}^{i} x_{i} U^{(0)}\left(x^{-}, x^{A}, x^{I}, \theta^{\dot{a}}, \bar{\theta}^{\dot{a}}, \lambda^{a}, \bar{\lambda}^{a}\right), \\
\frac{\partial}{\partial \kappa^{\dot{a}}} \bar{U}_{a}^{(1)} & =+\mathrm{i} E_{-}\left(\sigma^{i} \Pi\right)_{a \dot{a}} x_{i} U^{(0)}\left(x^{-}, x^{A}, x^{I}, \theta^{\dot{a}}, \bar{\theta}^{\dot{a}}, \lambda^{a}, \bar{\lambda}^{a}\right) .
\end{aligned}
$$

Note that $U_{\text {final }}$ has a well defined $\mathrm{e}_{+}$charge because of (3.50) and (5.24).

The super partners of $U$ can organized using a complex supersymmetry generator

$$
\tilde{\mathrm{q}}_{\dot{a}}=\mathrm{s}_{\dot{a}}+\mathrm{i} \Pi_{\dot{a} \dot{b}} \overline{\mathrm{s}}_{\dot{b}},
$$

and acting with $\tilde{\mathrm{q}}_{\dot{a}}$ on $U_{\text {final }}$ we generate the whole massless spectrum as described in 5 . The spectrum is then

$$
\begin{aligned}
& U(\phi)=\phi U_{\text {final }}\left(x^{-}, x^{+}, x^{A}, x^{I}, \theta^{\dot{a}}, \bar{\theta}^{\dot{a}}, \lambda^{a}, \bar{\lambda}^{a}\right), \\
& U\left(\phi^{\dot{a}}\right)=\phi^{\dot{a}} \tilde{\mathrm{q}}_{\dot{a}} U_{\text {final }}\left(x^{-}, x^{+}, x^{A}, x^{I}, \theta^{\dot{a}}, \bar{\theta}^{\dot{a}}, \lambda^{a}, \bar{\lambda}^{a}\right) \\
& U\left(\phi^{\dot{a} \dot{b}}\right)=\phi^{\dot{a} \dot{b}} \tilde{\mathrm{q}}_{\dot{b}} \tilde{\mathrm{q}}_{\dot{a}} U_{\text {final }}\left(x^{-}, x^{+}, x^{A}, x^{I}, \theta^{\dot{a}}, \bar{\theta}^{\dot{a}}, \lambda^{a}, \bar{\lambda}^{a}\right) \\
& U\left(\phi^{\dot{a} \dot{b} \dot{c}}\right)=\phi^{\dot{a} \dot{b} \dot{c}} \tilde{\mathrm{q}}_{\dot{c}} \tilde{\mathrm{q}}_{\dot{b}} \tilde{\mathrm{q}}_{\dot{a}} U_{\text {final }}\left(x^{-}, x^{+}, x^{A}, x^{I}, \theta^{\dot{a}}, \bar{\theta}^{\dot{a}}, \lambda^{a}, \bar{\lambda}^{a}\right) \\
& U\left(\phi^{\dot{a} \dot{b} \dot{c} \dot{d}}\right)=\phi^{\dot{a} \dot{b} \dot{c} \dot{d}} \tilde{\mathrm{q}}_{\dot{d}} \tilde{\mathrm{q}}_{\dot{c}} \tilde{\mathrm{q}}_{\dot{b}} \tilde{\mathrm{q}}_{\dot{a}} U_{\text {final }}\left(x^{-}, x^{+}, x^{A}, x^{I}, \theta^{\dot{a}}, \bar{\theta}^{\dot{a}}, \lambda^{a}, \bar{\lambda}^{a}\right) \\
& U\left(\tilde{\phi}_{\dot{a} \dot{b} \dot{c}}\right)=\tilde{\phi}_{\dot{a} \dot{b} \dot{c}} \epsilon^{\dot{a} \dot{b} \dot{b} \dot{c}_{1} \dot{a}_{2} \dot{a}_{3} \dot{a}_{4} \dot{a}_{5}} \tilde{\mathrm{q}}_{\dot{a}_{5}} \tilde{\mathrm{q}}_{\dot{q}_{4}} \tilde{\mathrm{q}}_{\dot{a}_{3}} \tilde{\mathrm{q}}_{\dot{a}_{2}} \tilde{\mathrm{q}}_{\dot{a}_{1}} U_{\text {final }}\left(x^{-}, x^{+}, x^{A}, x^{I}, \theta^{\dot{a}}, \bar{\theta}^{\dot{a}}, \lambda^{a}, \bar{\lambda}^{a}\right) \\
& U\left(\tilde{\phi}_{\dot{a} \dot{b}}\right)=\tilde{\phi}_{\dot{a} \dot{b}} \epsilon^{\dot{a} \dot{b} \dot{a}_{1} \dot{a}_{2} \dot{a}_{3} \dot{a}_{4} \dot{a}_{5} \dot{a}_{6}} \tilde{\mathrm{q}}_{\dot{a}_{6}} \tilde{\mathrm{q}}_{\dot{a}_{5}} \tilde{\mathrm{q}}_{\dot{a}_{4}} \tilde{\mathrm{q}}_{\dot{a}_{3}} \tilde{\mathrm{q}}_{\dot{a}_{2}} \tilde{\mathrm{q}}_{\dot{a}_{1}} U_{\text {final }}\left(x^{-}, x^{+}, x^{A}, x^{I}, \theta^{\dot{a}}, \bar{\theta}^{\dot{a}}, \lambda^{a}, \bar{\lambda}^{a}\right) \\
& U\left(\tilde{\phi}_{\dot{a}}\right)=\tilde{\phi}_{\dot{a}} \epsilon^{\dot{a} \dot{a}_{1} \dot{a}_{2} \dot{a}_{3} \dot{a}_{4} \dot{a}_{5} \dot{a}_{6} \dot{a}_{7}} \tilde{\mathrm{q}}_{\dot{a}_{7}} \tilde{\mathrm{q}}_{\dot{a}_{6}} \tilde{\mathrm{q}}_{\dot{a}_{5}} \tilde{\mathrm{q}}_{\dot{a}_{4}} \tilde{\mathrm{q}}_{\dot{a}_{3}} \tilde{\mathrm{q}}_{\dot{a}_{2}} \tilde{\mathrm{q}}_{\dot{a}_{1}} U_{\text {final }}\left(x^{-}, x^{+}, x^{A}, x^{I}, \theta^{\dot{a}}, \bar{\theta}^{\dot{a}}, \lambda^{a}, \bar{\lambda}^{a}\right) \\
& U(\tilde{\phi})=\tilde{\phi} \epsilon^{\dot{a}_{1} \dot{a}_{2} \dot{a}_{3} \dot{a}_{4} \dot{a}_{5} \dot{a}_{6} \dot{a}_{7} \dot{a}_{8}} \tilde{\mathrm{q}}_{\dot{a}_{8}} \tilde{\mathrm{q}}_{\dot{a}_{7}} \tilde{\mathrm{q}}_{\dot{a}_{6}} \tilde{\mathrm{q}}_{\dot{a}_{5}} \tilde{\mathrm{q}}_{\dot{a}_{4}} \tilde{\mathrm{q}}_{\dot{a}_{3}} \tilde{\mathrm{q}}_{\dot{a}_{2}} \tilde{\mathrm{q}}_{\dot{a}_{1}} U_{\text {final }}\left(x^{-}, x^{+}, x^{A}, x^{I}, \theta^{\dot{a}}, \bar{\theta}^{\dot{a}}, \lambda^{a}, \bar{\lambda}^{a}\right) \text {. }
\end{aligned}
$$


The set of polarizations $\left\{\phi, \phi^{\dot{a}}, \phi^{\dot{a} \dot{b}}, \phi^{\dot{a} \dot{b} \dot{c}}, \phi^{\dot{a} \dot{b} \dot{c} \dot{d}}, \tilde{\phi}_{\dot{a} \dot{b} \dot{c}}, \tilde{\phi}_{\dot{a} \dot{b}}, \tilde{\phi}_{\dot{a}}, \tilde{\phi}\right\}$ describe the 256 supergravity states. Notice that $\phi^{\dot{a} \dot{b} \dot{c} \dot{d}}$ is self-dual. The vertex operator $U_{\text {final }}\left(x^{-}, x^{+}, x^{A}, x^{I}, \theta^{\dot{a}}\right.$, $\left.\bar{\theta}^{\dot{a}}, \lambda^{a}, \bar{\lambda}^{a}\right)$ is the generating operator for whole massless spectrum. The value of $E_{+}$can be raised further using the bosonic creation operators. This will add extra dependence on the bosonic coordinates in the form of Hermite polynomials.

\section{$6 \quad$ Integrated vertex operator}

We will start this section with the most general unintegrated vertex operator $U=\lambda^{\alpha} \bar{\lambda}^{\beta} A_{\alpha \beta}$ satisfying $Q U=0$ which implies

$$
\nabla_{(\alpha} A_{\beta) \bar{\gamma}}=\mathrm{i} \gamma_{\alpha \beta}^{\underline{a}} A_{\underline{a} \gamma}, \quad \bar{\nabla}_{(\alpha} A_{\gamma \beta)}=\mathrm{i} \gamma_{\alpha \beta}^{\underline{a}} A_{\gamma \underline{a}} .
$$

The invariance implied by $\delta U=Q \Lambda$ gives

$$
\delta A_{\alpha \bar{\beta}}=\nabla_{\alpha} \bar{\Lambda}_{\beta}+\bar{\nabla}_{\beta} \Lambda_{\alpha}
$$

where the gauge parameters satisfy

$$
\nabla_{(\alpha} \Lambda_{\beta)}=\mathrm{i} \gamma{ }_{\alpha \beta}^{\underline{a}} \Lambda_{\underline{a}}, \quad \bar{\nabla}_{(\alpha} \bar{\Lambda}_{\beta)}=\mathrm{i} \gamma \frac{a}{\alpha \beta} \bar{\Lambda}_{\underline{a}} .
$$

In our case, we use light-cone coordinates. It turns out that the physical degrees of freedom in $U$ are in $A_{a b}$, then the other components have to be gauge fixed using (6.2). Let us check this. Consider the equation for $A_{\dot{a} \bar{\gamma}}$

$$
\nabla_{(\dot{a}} A_{\dot{b}) \bar{\gamma}}=\mathrm{i} \delta_{\dot{a} \dot{b}} A_{-\bar{\gamma}} .
$$

If there exist a solution of this equation of the form (6.2), then we can use this gauge symmetry to put $A_{\dot{a} \bar{\gamma}}$ to zero. Try $A_{\dot{a} \bar{\gamma}}=\nabla_{\dot{a}} \bar{\Lambda}_{\bar{\gamma}}+\nabla_{\bar{\gamma}} \Lambda_{\dot{a}}$ and find the gauge parameters that solve (6.4). We obtain

$$
\nabla_{(\dot{a}} A_{\dot{b}) \bar{\gamma}}=\left\{\nabla_{\dot{a}}, \nabla_{\dot{b}}\right\}+\nabla_{(\dot{a}} \nabla_{\bar{\gamma}} \Lambda_{\dot{b})}=-T_{\dot{a} \dot{b}}{ }^{-} \nabla_{-} \bar{\Lambda}_{\bar{\gamma}}-\nabla_{\bar{\gamma}} \nabla_{(\dot{a}} \Lambda_{\dot{b})}+\left\{\nabla_{\bar{\gamma}}, \nabla_{(\dot{a}}\right\} \Lambda_{\dot{b})} .
$$

Using the values of torsion and (6.3)

$$
\nabla_{(\dot{a}} A_{\dot{b}) \bar{\gamma}}=\mathrm{i} \delta_{\dot{a} \dot{b}}\left(\nabla_{-} \bar{\Lambda}_{\bar{\gamma}}-\nabla_{\bar{\gamma}} \Lambda_{-}\right)+\left\{\nabla_{\bar{\gamma}}, \nabla_{(\dot{a}}\right\} \Lambda_{\dot{b})}
$$

The last anti-commutator is a curvature. It vanishes for $\bar{\gamma}=\dot{\bar{c}}$. For $\bar{\gamma}=\bar{c}$, it is proportional

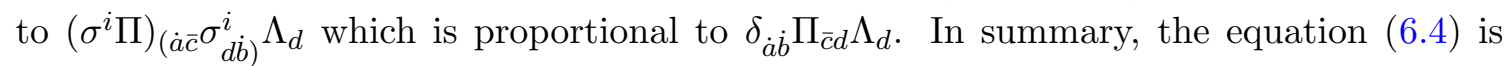
satisfied by a gauge transformation expression. Then, $A_{\dot{a} \bar{\gamma}}$ can be gauge fixed to zero. Similarly, $A_{\gamma \dot{\bar{a}}}$ can be put to zero.

In the gauge $A_{\dot{a} \bar{\gamma}}=A_{\gamma \dot{\bar{a}}}=0$, the unintegrated vertex operator is $U=\lambda^{a} \bar{\lambda}^{b} A_{a b}$ and the superfield $A_{a b}$ satisfies the equations

$$
\nabla_{(a} A_{b) c}=\mathrm{i} \delta_{a b} A_{+c}, \quad \nabla_{\dot{a}} A_{b c}=\mathrm{i} \sigma_{b \dot{a}}^{i} A_{i c}, \quad \bar{\nabla}_{(a} A_{c b)}=\mathrm{i} \delta_{a b} A_{c+}, \quad \bar{\nabla}_{\dot{a}} A_{c b}=\mathrm{i} \sigma_{b \dot{a}}^{i} A_{c i}
$$


From here, the second fermionic covariant derivatives of $A_{a b}$ are constrained to satisfy

$$
\begin{aligned}
& \nabla_{a} A_{+c}-\nabla_{+} A_{a c}=0, \quad \nabla_{-} A_{a c}=2 W_{a c}, \quad \nabla_{a} A_{i c}-\nabla_{i} A_{a c}=\sigma_{a \dot{b}}^{i} W_{\dot{b} c}, \\
& \nabla_{\dot{a}} A_{+c}=-2 W_{\dot{a} c}, \quad \nabla_{\dot{a}} A_{i c}=\sigma_{b \dot{a}}^{i} W_{b c}, \\
& \bar{\nabla}_{a} A_{c+}-\nabla_{+} A_{c a}=0, \quad \nabla_{-} A_{c a}=2 \bar{W}_{c a}, \quad \bar{\nabla}_{a} A_{c i}-\nabla_{i} A_{c a}=\sigma_{a \dot{a}}^{i} \bar{W}_{c \dot{b}} \\
& \bar{\nabla}_{\dot{a}} A_{c+}=-2 \bar{W}_{c \dot{a}}, \quad \bar{\nabla}_{\dot{a}} A_{c i}=\sigma_{b \dot{a}}^{i} \bar{W}_{c b} .
\end{aligned}
$$

Note that $W_{a b}=\bar{W}_{a b}$. The next group of equations come from performing the third fermionic covariant derivative of $A_{a b}$. These equations depend on the field strengths $F_{\underline{a} \underline{ } \gamma}=$ $\nabla_{[\underline{a}} A_{\underline{b}] \gamma}$ and $F_{\gamma \underline{a b}}=\nabla_{[\underline{a}} A_{\gamma \underline{b}]}$ and they are

$$
\begin{aligned}
\nabla_{a} W_{b c} & =-\frac{\mathrm{i}}{4} \delta_{a b} F_{+-c}+\frac{\mathrm{i}}{4}\left(\sigma_{i j}\right)_{a b} F_{i j c}, & \nabla_{\dot{a}} W_{b c} & =\frac{\mathrm{i}}{2} \sigma_{b \dot{a}}^{i} F_{-i c}, \\
\nabla_{a} W_{\dot{b} c}-\frac{1}{2} \Pi_{\dot{b} \dot{c}} \bar{\nabla}_{\dot{c}} A_{a c} & =\frac{\mathrm{i}}{2} \sigma_{a \dot{b}}^{i} F_{+i c}, & \nabla_{\dot{a}} W_{\dot{b} c} & =\frac{\mathrm{i}}{4} \delta_{\dot{a} \dot{b}} F_{+-c}+\frac{\mathrm{i}}{4}\left(\sigma_{i j}\right)_{\dot{a} \dot{b}} F_{i j c}, \\
\bar{\nabla}_{a} W_{c b} & =-\frac{\mathrm{i}}{4} \delta_{a b} F_{c+-}+\frac{\mathrm{i}}{4}\left(\sigma_{i j}\right)_{a b} F_{c i j}, & \bar{\nabla}_{\dot{a}} W_{c b} & =\frac{\mathrm{i}}{2} \sigma_{b \dot{a}}^{i} F_{c-i}, \\
\bar{\nabla}_{a} \bar{W}_{c \dot{b}}+\frac{1}{2} \Pi_{\dot{b} \dot{c}} \nabla_{\dot{c}} A_{c a} & =\frac{\mathrm{i}}{2} \sigma_{a \dot{b}}^{i} F_{c+i}, & \bar{\nabla}_{\dot{a}} \bar{W}_{c \dot{b}} & =\frac{\mathrm{i}}{4} \delta_{\dot{a} \dot{b}} F_{c+-}+\frac{\mathrm{i}}{4}\left(\sigma_{i j}\right)_{\dot{a} \dot{b}} F_{c i j} .
\end{aligned}
$$

As in flat space, the integrated vertex operator $\mathcal{V}$ is obtained from the equations

$$
Q \mathcal{V}=\partial \overline{\mathcal{W}}-\bar{\partial} \mathcal{W}, \quad Q \overline{\mathcal{W}}=\bar{\partial} U, \quad Q \mathcal{W}=\partial U .
$$

Note that we can use the equations of motion derived from the action (4.2). The equations we need are

$$
\bar{J}_{1}^{a}=J_{3}^{a}=\bar{\nabla} \lambda^{a}=\nabla \bar{\lambda}^{a}=0, \quad \bar{J}_{1}^{\dot{a}}=-\frac{\mathrm{i}}{2} \Pi^{\dot{a} \dot{b}} \bar{d}_{\dot{b}}, \quad J_{3}^{\dot{a}}=\frac{\mathrm{i}}{2} \Pi^{\dot{a} \dot{b}} d_{\dot{b}} .
$$

The $\mathcal{W}$ satisfying $Q \mathcal{W}=\partial U$ is given by

$$
\begin{aligned}
\mathcal{W}=\bar{\lambda}^{b}( & J_{1}^{a} A_{a b}+J^{+} A_{+b}+J^{i} A_{i b}-\mathrm{i} d_{a} W_{a b}-\mathrm{i} d_{\dot{a}} W_{\dot{a} b} \\
& \left.+N^{+-} F_{+-b}+N^{+i} F_{+i b}+N^{-i} F_{-i b}+\frac{1}{2} N^{i j} F_{i j b}\right) \equiv \bar{\lambda}^{b}\left(J_{1}^{a} A_{a b}+\varphi_{b}\right),
\end{aligned}
$$

where $\varphi_{b}$ is defined by (6.12) and

$$
\begin{aligned}
N^{+-} & =-\frac{1}{4} \lambda^{a} \omega_{a}+\frac{1}{4} \lambda^{\dot{a}} \omega_{\dot{a}}, & N^{+i} & =\frac{1}{2} \sigma_{a \dot{b}}^{i} \lambda^{a} \omega_{\dot{b}}, \\
N^{-i} & =\frac{1}{2} \sigma_{a \dot{b}}^{i} \lambda^{\dot{b}} \omega_{a}, & N^{i j} & =\frac{1}{2} \lambda^{a} \omega_{b} \sigma_{a b}^{i j}+\frac{1}{2} \lambda^{\dot{a}} \omega_{\dot{b}} \sigma_{\dot{a} \dot{b}}^{i j} .
\end{aligned}
$$

The BRST transformations are $Q \omega_{a}=d_{a}, Q \omega_{\dot{a}}=d_{\dot{a}}$ and

$$
\begin{aligned}
Q J^{a} & =\nabla \lambda^{a}, \quad Q J^{+}=\mathrm{i}\left(\lambda^{a} J_{1}^{a}+\bar{\lambda}^{a} J_{3}^{a}\right), \quad Q J^{i}=\mathrm{i} \sigma_{a \dot{b}}^{i}\left(\lambda^{(a} J_{1}^{b \dot{b})}+\bar{\lambda}^{(a} J_{3}^{\dot{b})}\right), \\
Q d_{a} & =\mathrm{i}\left(2 \lambda_{a} J^{-}-\sigma_{a \dot{b}}^{i} \lambda^{\dot{b}} J^{i}\right)-\frac{\mathrm{i}}{2}\left(\sigma_{[i j]} \Pi\right)_{a b} \bar{\lambda}^{b} N^{[i j]}+\mathrm{i}\left(\sigma_{i} \Pi\right)_{a \dot{b}} \bar{\lambda}^{\dot{b}} N^{+i}, \\
Q d_{\dot{a}} & =\mathrm{i}\left(2 \lambda^{\dot{a}} J^{+}-\sigma_{b \dot{a}}^{i} \lambda^{b} J^{i}\right)-\mathrm{i}\left(\sigma_{i} \Pi\right)_{b \dot{a}} \bar{\lambda}^{b} N^{+i} .
\end{aligned}
$$


Consider the BRST transformation of the first term in (6.12). It contains $\bar{\lambda}^{b} \nabla \lambda^{a} A_{a b}$ which is equal to $\nabla\left(\bar{\lambda}^{b} \lambda^{a} A_{a b}\right)-\bar{\lambda}^{b} \lambda^{a} \nabla A_{a b}=\nabla U-\bar{\lambda}^{b} \lambda^{a} \nabla A_{a b}$. Here we are using the equations of motion (6.11). Then

$$
\begin{aligned}
Q \mathcal{W}= & \partial U+\bar{\lambda}^{b}\left(-\lambda^{a}\left[J_{1}^{c} \nabla_{c} A_{a b}+J_{1}^{\dot{c}} \nabla_{\dot{c}} A_{a b}+J_{3}^{\dot{c}} \bar{\nabla}_{\dot{c}} A_{a b}+J^{+} \nabla_{+} A_{a b}+J^{-} \nabla_{-} A_{a b}+J^{i} A_{a b}\right]\right. \\
& -J_{1}^{a}\left[\lambda^{c} \nabla_{c} A_{a b}+\lambda^{\dot{c}} \nabla_{\dot{c}} A_{a b}+\bar{\lambda}^{c} \bar{\nabla}_{c} A_{a b}+\bar{\lambda}^{\dot{c}} \bar{\nabla}_{\dot{c}} A_{a b}\right]+\mathrm{i}\left[\lambda^{c} J_{1}^{c}\right] A_{+b} \\
& +J^{+}\left[\lambda^{c} \nabla_{c} A_{+b}+\lambda^{\dot{c}} \nabla_{\dot{c}} A_{+b}+\bar{\lambda}^{c} \bar{\nabla}_{c} A_{+b}+\bar{\lambda}^{\dot{c}} \bar{\nabla}_{\dot{c}} A_{+b}\right]+\mathrm{i} \sigma_{c \dot{d}}^{i}\left[\lambda^{c} J_{1}^{\dot{d}}+\lambda^{\dot{d}} J_{1}^{c}+\bar{\lambda}^{c} J_{3}^{\dot{d}}\right] A_{i b} \\
& +J^{i}\left[\lambda^{c} \nabla_{c} A_{i b}+\lambda^{\dot{c}} \nabla_{\dot{c}} A_{i b}+\bar{\lambda}^{c} \bar{\nabla}_{c} A_{i b}+\bar{\lambda}^{\dot{c}} \bar{\nabla}_{\dot{c}} A_{i b}\right] \\
& +\left[2 \lambda^{a} J^{-}-\sigma_{a \dot{c}}^{i} \lambda^{\dot{c}} J^{i}-\frac{1}{2}\left(\sigma_{[i j]} \Pi\right)_{a c} \bar{\lambda}^{c} N^{[i j]}+\left(\sigma_{i} \Pi\right)_{a \dot{c}} \bar{\lambda}^{\dot{c}} N^{+i}\right] W_{a b} \\
& +\mathrm{i} d_{a}\left[\lambda^{c} \nabla_{c} W_{a b}+\lambda^{\dot{c}} \nabla_{\dot{c}} W_{a b}+\bar{\lambda}^{c} \bar{\nabla}_{c} W_{a b}+\bar{\lambda}^{\dot{c}} \bar{\nabla}_{\dot{c}} W_{a b}\right] \\
& +\left[2 \lambda^{\dot{a}} J^{+}-\sigma_{c \dot{a}}^{i} \lambda^{c} J^{i}-\left(\sigma_{i} \Pi\right)_{c \dot{a}} \bar{\lambda}^{c} N^{+i}\right] W_{\dot{a} b} \\
& +\mathrm{i} d_{\dot{a}}\left[\lambda^{c} \nabla_{c} W_{\dot{a} b}+\lambda^{\dot{c}} \nabla_{\dot{c}} W_{\dot{a} b}+\bar{\lambda}^{c} \bar{\nabla}_{c} W_{\dot{a} b}+\bar{\lambda}^{\dot{c}} \bar{\nabla}_{\dot{c}} W_{\dot{a} b}\right]+\frac{1}{4}\left[\lambda^{a} d_{b} \sigma_{a b}^{i j}+\lambda^{\dot{a}} d_{\dot{b}} \sigma_{\dot{a} \dot{b}}^{i j}\right] F_{i j b} \\
& +\frac{1}{2} N^{i j}\left[\lambda^{c} \nabla_{c} F_{i j b}+\lambda^{\dot{c}} \nabla_{\dot{c}} F_{i j b}+\bar{\lambda}^{c} \bar{\nabla}_{c} F_{i j b}+\bar{\lambda}^{\dot{c}} \bar{\nabla}_{\dot{c}} F_{i j b}\right]+\frac{1}{4}\left[-\lambda^{a} d_{a}+\lambda^{\dot{a}} d_{\dot{a}}\right] F_{+-b} \\
& +N^{+-}\left[\lambda^{c} \nabla_{c} F_{+-b}+\lambda^{\dot{c}} \nabla_{\dot{c}} F_{+-b}+\bar{\lambda}^{c} \bar{\nabla}_{c} F_{+-b}+\bar{\lambda}^{\dot{c}} \bar{\nabla}_{\dot{c}} F_{+-b}\right]+\frac{1}{2}\left[\sigma_{a \dot{b}}^{i} \lambda^{a} d_{\dot{b}}\right] F_{+i b} \\
& N^{+i}\left[\lambda^{c} \nabla_{c} F_{+i b}+\lambda^{\dot{c}} \nabla_{\dot{c}} F_{+i b}+\bar{\lambda}^{c} \bar{\nabla}_{c} F_{+i b}+\bar{\lambda}^{\dot{c}} \bar{\nabla}_{\dot{c}} F_{+i b}\right]+\frac{1}{2}\left[\sigma_{a \dot{b}}^{i} \lambda^{\dot{b}} d_{a}\right] F_{-i b} \\
& \left.+N^{-i}\left[\lambda^{c} \nabla_{c} F_{-i b}+\lambda^{\dot{c}} \nabla_{\dot{c}} F_{-i b}+\bar{\lambda}^{c} \bar{\nabla}_{c} F_{-i b}+\bar{\lambda}^{\dot{c}} \bar{\nabla}_{\dot{c}} F_{-i b}\right]\right) .
\end{aligned}
$$

Here we used the equation of motion $J_{3}^{a}=0$. It is easy to check that the terms with $\lambda^{\alpha} J_{1}^{\beta}$ and are zero because the equations (6.7). The term with $\lambda^{a} J_{3}^{\dot{a}}$ mixes with terms with $\lambda^{a} d_{\dot{a}}$, because the last equation in (6.11), and the result is one of the equations in (6.9). The terms with $\lambda^{\alpha} J_{2}$ also vanish because the equations (6.8). Similarly, the terms involving $\lambda^{\alpha} d_{\beta}$ also vanish. They factor the equations (6.9). Up to now, we are left with terms with $\omega_{\alpha}$ and terms quadratic in $\bar{\lambda}$. They are

$$
\begin{aligned}
& \bar{\lambda}^{b}\left(-J_{1}^{a}\left[\bar{\lambda}^{c} \bar{\nabla}_{c} A_{a b}+\bar{\lambda}^{\dot{c}} \bar{\nabla}_{\dot{c}} A_{a b}\right]+J^{+}\left[\bar{\lambda}^{c} \bar{\nabla}_{c} A_{+b}+\bar{\lambda}^{\dot{c}} \bar{\nabla}_{\dot{c}} A_{+b}\right]+\mathrm{i} \sigma_{c \dot{d}}^{i}\left[\bar{\lambda}^{c} J_{3}^{\dot{d}}\right] A_{i b}\right. \\
& +J^{i}\left[\bar{\lambda}^{c} \bar{\nabla}_{c} A_{i b}+\bar{\lambda}^{\dot{c}} \bar{\nabla}_{\dot{c}} A_{i b}\right]+\left[-\frac{1}{2}\left(\sigma_{[i j]} \Pi\right)_{a c} \bar{\lambda}^{c} N^{[i j]}+\left(\sigma_{i} \Pi\right)_{a \dot{c}} \bar{\lambda}^{\dot{c}} N^{+i}\right] W_{a b} \\
& +\mathrm{i} d_{a}\left[\bar{\lambda}^{c} \bar{\nabla}_{c} W_{a b}+\bar{\lambda}^{\dot{c}} \bar{\nabla}_{\dot{c}} W_{a b}\right]+\left[-\left(\sigma_{i} \Pi\right)_{c \dot{a}} \bar{\lambda}^{c} N^{+i}\right] W_{\dot{a} b}+\mathrm{i} d_{\dot{a}}\left[\bar{\lambda}^{c} \bar{\nabla}_{c} W_{\dot{a} b}+\bar{\lambda}^{\dot{c}} \bar{\nabla}_{\dot{c}} W_{\dot{a} b}\right] \\
& +\frac{1}{2} N^{i j}\left[\lambda^{c} \nabla_{c} F_{i j b}+\lambda^{\dot{c}} \nabla_{\dot{c}} F_{i j b}+\bar{\lambda}^{c} \bar{\nabla}_{c} F_{i j b}+\bar{\lambda}^{\dot{c}} \bar{\nabla}_{\dot{c}} F_{i j b}\right] \\
& +N^{+-}\left[\lambda^{c} \nabla_{c} F_{+-b}+\lambda^{\dot{c}} \nabla_{\dot{c}} F_{+-b}+\bar{\lambda}^{c} \bar{\nabla}_{c} F_{+-b}+\bar{\lambda}^{\dot{c}} \bar{\nabla}_{\dot{c}} F_{+-b}\right] \\
& +N^{+i}\left[\lambda^{c} \nabla_{c} F_{+i b}+\lambda^{\dot{c}} \nabla_{\dot{c}} F_{+i b}+\bar{\lambda}^{c} \bar{\nabla}_{c} F_{+i b}+\bar{\lambda}^{\dot{c}} \bar{\nabla}_{\dot{c}} F_{+i b}\right] \\
& \left.+N^{-i}\left[\lambda^{c} \nabla_{c} F_{-i b}+\lambda^{\dot{c}} \nabla_{\dot{c}} F_{-i b}+\bar{\lambda}^{c} \bar{\nabla}_{c} F_{-i b}+\bar{\lambda}^{\dot{c}} \bar{\nabla}_{\dot{c}} F_{-i b}\right]\right) .
\end{aligned}
$$

The terms quadratic in $\bar{\lambda}$ will vanish because of the pure spinor conditions $\bar{\lambda}^{a} \bar{\lambda}^{a}=\sigma_{a \dot{b}}^{i} \bar{\lambda}^{a} \bar{\lambda}^{\dot{b}}=$ 0 and the commutation relations $\left[\nabla_{A}, \nabla_{B}\right]$ which are given by the torsion and curvature of the plane wave background. These kind of terms with $J_{1}^{a}$ in (6.16) zero because of (6.7). 
There are two terms with $J^{+}$, the first goes with

$$
\begin{aligned}
& \bar{\lambda}^{b} \bar{\lambda}^{c} \bar{\nabla}_{c} A_{+b}=-\frac{\mathrm{i}}{8} \bar{\lambda}^{b} \bar{\lambda}^{c} \bar{\nabla}_{c} \nabla_{d} A_{d b}=\frac{\mathrm{i}}{8} \bar{\lambda}^{b} \bar{\lambda}^{c} \nabla_{d} \bar{\nabla}_{c} A_{d b}=0 \\
& \frac{\mathrm{i}}{16} \bar{\lambda}^{b} \bar{\lambda}^{c} \nabla_{d} \bar{\nabla}_{(c} A_{d b)}=-\frac{1}{16} \bar{\lambda}^{b} \bar{\lambda}^{b} \nabla_{d} A_{d+}=0 .
\end{aligned}
$$

Similarly, the second term with $J^{+}$and the terms with $J^{i}$ also vanish. The last term in the first line of (6.16) combines with the term involving $\bar{\lambda}^{c}$ in the last term of the third line of (6.16). They go with

$$
\bar{\lambda}^{b} \bar{\lambda}^{c}\left(\bar{\nabla}_{c} W_{\dot{a} b}+\frac{\mathrm{i}}{2}\left(\sigma^{i} \Pi\right)_{c \dot{a}} A_{i b}\right)
$$

but

$$
\bar{\nabla}_{c} W_{\dot{a} b}=-\frac{1}{2} \bar{\nabla}_{c} \nabla_{\dot{a}} A_{+b}=-\frac{1}{2}\left\{\nabla_{\dot{a}}, \bar{\nabla}_{c}\right\} A_{+b}+\frac{1}{2} \nabla_{\dot{a}} \bar{\nabla}_{c} A_{+b}
$$

Note that the last term will vanish in (6.19) because of (6.18). Using the non-vanishing curvature component for the commutator are $R_{\dot{a} c+}{ }^{i}=-\mathrm{i}\left(\sigma^{i} \Pi\right)_{c \dot{a}}$ and $R_{\dot{a} c b} \dot{d}=-\frac{\dot{i}}{2} \sigma_{b \dot{d}}^{i}\left(\sigma^{i} \Pi\right)_{c \dot{a}}$ we obtain that

$$
\bar{\nabla}_{c} W_{\dot{a} b}=-\frac{\mathrm{i}}{2}\left(\sigma^{i} \Pi\right)_{c \dot{a}} A_{i b}-\frac{\mathrm{i}}{4} \sigma_{b \dot{d}}^{i}\left(\sigma^{i} \Pi\right)_{c \dot{a}} A_{+\dot{d}}
$$

but the last term vanishes in (6.19) because $\bar{\lambda}^{b} \bar{\lambda}^{c} \sigma_{b \dot{c}}^{i} \sigma_{c \dot{d}}^{i}=0$. The first term here cancel the second term in (6.19). Then, we have verified that (6.19) is zero. The terms with $d_{\alpha}$ in (6.16) are also zero because (6.9) and pure spinor conditions. Up to this point we have terms with $\omega_{\alpha}$, which are

$$
\begin{aligned}
& \bar{\lambda}^{b}\left(\left[-\frac{1}{2}\left(\sigma_{[i j]} \Pi\right)_{a c} \bar{\lambda}^{c} N^{[i j]}+\left(\sigma_{i} \Pi\right)_{a \dot{c}} \bar{\lambda}^{\dot{c}} N^{+i}\right] W_{a b}+\left[-\left(\sigma_{i} \Pi\right)_{c \dot{a}} \bar{\lambda}^{c} N^{+i}\right] W_{\dot{a} b}\right. \\
& \quad+\frac{1}{2} N^{i j}\left[\lambda^{c} \nabla_{c} F_{i j b}+\lambda^{\dot{c}} \nabla_{\dot{c}} F_{i j b}+\bar{\lambda}^{c} \bar{\nabla}_{c} F_{i j b}+\bar{\lambda}^{\dot{c}} \bar{\nabla}_{\dot{c}} F_{i j b}\right] \\
& \quad+N^{+-}\left[\lambda^{c} \nabla_{c} F_{+-b}+\lambda^{\dot{c}} \nabla_{\dot{c}} F_{+-b}+\bar{\lambda}^{c} \bar{\nabla}_{c} F_{+-b}+\bar{\lambda}^{\dot{c}} \bar{\nabla}_{\dot{c}} F_{+-b}\right] \\
& \quad+N^{+i}\left[\lambda^{c} \nabla_{c} F_{+i b}+\lambda^{\dot{c}} \nabla_{\dot{c}} F_{+i b}+\bar{\lambda}^{c} \bar{\nabla}_{c} F_{+i b}+\bar{\lambda}^{c} \bar{\nabla}_{\dot{c}} F_{+i b}\right] \\
& \left.\quad+N^{-i}\left[\lambda^{c} \nabla_{c} F_{-i b}+\lambda^{\dot{c}} \nabla_{\dot{c}} F_{-i b}+\bar{\lambda}^{c} \bar{\nabla}_{c} F_{-i b}+\bar{\lambda}^{\dot{c}} \bar{\nabla}_{\dot{c}} F_{-i b}\right]\right) .
\end{aligned}
$$

The terms involving $\lambda^{\alpha}$ have the form $N^{\underline{a b}} \lambda^{\alpha} \nabla_{\alpha} F_{\underline{a b} \beta}$. Because of the pure spinor condition, this expression becomes proportional to the equation of motion for $W^{\alpha}{ }_{\beta}$ (see section 4 of [31]). That remains are quadratic in $\bar{\lambda}$, the part with $F$ had the form $\bar{\lambda}^{\beta} \bar{\lambda}^{\alpha} \bar{\nabla}_{\alpha} F_{\underline{a b} \beta}$. This expression combines with the first line in (6.22) to vanish (see again section 4 of [31]). A similar calculation gives $\overline{\mathcal{W}}$, satisfying $Q \overline{\mathcal{W}}=\bar{\partial} U$, given by

$$
\begin{aligned}
\overline{\mathcal{W}}=\lambda^{a}( & \bar{J}_{3}^{b} A_{a b}+\bar{J}^{+} A_{a+}+\bar{J}^{i} A_{a i}-\mathrm{i} \bar{d}_{b} W_{a b}-\mathrm{i} \bar{d}_{\dot{b}} \bar{W}_{a \dot{b}} \\
& \left.+\bar{N}^{+-} F_{a+-}+\bar{N}^{+i} F_{a+i}+\bar{N}^{-i} F_{a-i}+\frac{1}{2} \bar{N}^{i j} F_{a i j}\right) \equiv \lambda^{a}\left(\bar{J}_{3}^{b} A_{a b}+\bar{\varphi}_{a}\right),
\end{aligned}
$$


where

$$
\begin{aligned}
\bar{N}^{+-} & =-\frac{1}{4} \bar{\lambda}^{a} \bar{\omega}_{a}+\frac{1}{4} \bar{\lambda}^{\dot{a}} \bar{\omega}_{\dot{a}}, & \bar{N}^{+i} & =\frac{1}{2} \sigma_{a \dot{b}}^{i} \bar{\lambda}^{a} \bar{\omega}_{\dot{b}}, \\
\bar{N}^{-i} & =\frac{1}{2} \sigma_{a \dot{b}}^{i} \bar{\lambda}^{b} \bar{\omega}_{a}, & \bar{N}^{i j} & =\frac{1}{2} \bar{\lambda}^{a} \bar{\omega}_{b} \sigma_{a b}^{i j}+\frac{1}{2} \bar{\lambda}^{\dot{a}} \bar{\omega}_{\dot{b}} \sigma_{\dot{a} \dot{b}}^{i j} .
\end{aligned}
$$

A further simplification appears if we use the $x$-dependence of the superfields in the form $e^{\mathrm{i} E_{-} x^{-}-\frac{E_{-}}{4} x^{2}}$. We have that

$$
\begin{gathered}
\mathcal{W}=\bar{\lambda}^{b}\left(\left[J_{1}^{a}+\frac{E_{-}}{2} d_{a}-\frac{E_{-}}{4} d_{\dot{a}} \sigma_{a \dot{a}}^{i} x^{i}\right] A_{a b}+\left[J^{+}-\mathrm{i} E_{-} N^{+-}+\frac{E_{-}}{2} N^{+i} x^{i}\right] A_{+b}\right. \\
\left.+\left[J^{i}+\mathrm{i} E_{-} N^{-i}+\frac{E_{-}}{2} N^{i j} x^{j}\right] A_{i b}+N^{+i} \nabla_{+} A_{i b}\right) \\
\overline{\mathcal{W}}=\lambda^{a}\left(\left[\bar{J}_{3}^{b}+\frac{E_{-}}{2} \bar{d}_{b}-\frac{E_{-}}{4} \bar{d}_{\dot{b}} \sigma_{b \dot{b}}^{i} x^{i}\right] A_{a b}+\left[\bar{J}^{+}-\mathrm{i} E_{-} \bar{N}^{+-}+\frac{E_{-}}{4} \bar{N}^{+i} x^{i}\right] A_{a+}\right. \\
\left.+\left[\bar{J}^{i}+\mathrm{i} E_{-} \bar{N}^{-i}+\frac{E_{-}}{2} \bar{N}^{i j} x^{j}\right] A_{a i}+\bar{N}^{+i} \nabla_{+} A_{a i}\right) .
\end{gathered}
$$

The integrated vertex $\mathcal{V}$ is computed, as in flat space, by satisfying (6.10). In order to write a shorter expression we use in some terms of $\mathcal{V}$ a ten-dimensional covariant notation with a vector index $\underline{a}=(+,-, i)$ and a spinor index $\alpha=(a, \dot{a})$. The integrated is given by

$$
\begin{aligned}
\mathcal{V}= & J_{1}^{a} \bar{J}_{3}^{b} A_{a b}+J_{1}^{a} \bar{\varphi}_{a}-\bar{J}_{3}^{a} \varphi_{a}+J^{\underline{a}} \bar{J}^{\underline{b}} A_{\underline{a b}}+d_{\alpha} \bar{J} \underline{E}_{\underline{a}}{ }^{\alpha}+J^{a} \bar{d}_{\alpha} \bar{E}_{\underline{a}}{ }^{\alpha}+J^{\underline{c}} \bar{N}^{\underline{a b}} \Omega_{\underline{c a b}} \\
& +N^{\underline{a b}} \bar{J} \underline{\Omega^{c a b}} \bar{\Omega}_{\underline{\alpha}}+d_{\alpha} \bar{d}_{\beta} P^{\alpha \beta}+d_{\alpha} \bar{N}^{\underline{a b}} C_{\underline{a b}}{ }^{\alpha}+N^{\underline{a b}} \bar{d}_{\alpha} \bar{C}_{\underline{a b}}{ }^{\alpha}+N^{\underline{a b}} \bar{N}^{\underline{c d}} R_{\underline{a b c d}},
\end{aligned}
$$

where $\varphi_{a}$ is defined in (6.12) and $\bar{\varphi}_{a}$ is defined in (6.23). The following is a summary of the results. After imposing (6.10), the non-zero components of $A$ satisfies

$$
\begin{aligned}
& \nabla_{(a} A_{b)+}=\mathrm{i} \delta_{a b} A_{++}, \quad \bar{\nabla}_{(a} A_{+b)}=-\mathrm{i} \delta_{a b} A_{++}, \quad \nabla_{(a} A_{b) i}=\mathrm{i} \delta_{a b} A_{+i}, \quad \bar{\nabla}_{\dot{a}} A_{+a}=-\mathrm{i} \sigma_{a \dot{a}}^{i} A_{+i}, \\
& \bar{\nabla}_{(a} A_{i b)}=-\mathrm{i} \delta_{a b} A_{i+}, \quad \nabla_{\dot{a}} A_{a+}=\mathrm{i} \sigma_{a \dot{a}}^{i} A_{i+}, \quad \nabla_{\dot{a}} A_{a i}=\mathrm{i} \sigma_{a \dot{a}}^{j} A_{j i}, \quad \bar{\nabla}_{\dot{a}} A_{i a}=-\mathrm{i} \sigma_{a \dot{a}}^{j} A_{i j} .
\end{aligned}
$$

The non-zero components of $E$ satisfy

$$
E_{+a}=-\frac{\mathrm{i}}{2} \nabla_{-} A_{a+}, \quad E_{i a}=-\frac{\mathrm{i}}{2} \nabla_{-} A_{a i} .
$$

The non-zero components of $\bar{E}$ satisfy

$$
\bar{E}_{+a}=\frac{\mathrm{i}}{2} \nabla_{-} A_{+a}, \quad \bar{E}_{i a}=\frac{\mathrm{i}}{2} \nabla_{-} A_{i a}, \quad \bar{E}_{+\dot{a}}=\frac{\mathrm{i}}{2} \bar{\nabla}_{\dot{a}} A_{++}, \quad \bar{E}_{i \dot{a}}=\frac{\mathrm{i}}{2} \bar{\nabla}_{\dot{a}} A_{i+} .
$$

The non-zero components of $\Omega$ are

$$
\begin{aligned}
\Omega_{++-} & =-\frac{1}{2} \bar{\nabla}_{a} \bar{E}_{+a}, \quad \Omega_{i+-}=\frac{1}{2} \bar{\nabla}_{a} \bar{E}_{i a}, & \Omega_{++i} & =\frac{1}{4}\left(\sigma_{i}\right)_{a \dot{a}} \bar{\nabla}_{a} \bar{E}_{+\dot{a}}+\frac{\mathrm{i}}{8}\left(\sigma_{i} \Pi\right)_{a \dot{a}} \nabla_{\dot{a}} A_{+a}, \\
\Omega_{j+i} & =\frac{1}{4}\left(\sigma_{i}\right)_{a \dot{a}} \bar{\nabla}_{a} \bar{E}_{i \dot{a}}+\frac{\mathrm{i}}{8}\left(\sigma_{j} \Pi\right)_{a \dot{a}} \nabla_{\dot{a}} A_{i a}, & \Omega_{+-i} & =\frac{1}{4}\left(\sigma_{i}\right)_{a \dot{a}} \bar{\nabla}_{\dot{a}} \bar{E}_{+a}, \quad \Omega_{j-i}=\frac{1}{4}\left(\sigma_{i}\right)_{a \dot{a}} \bar{\nabla}_{\dot{a}} \bar{E}_{j a} \\
\Omega_{+i j} & =\frac{1}{8}\left(\sigma_{i j}\right)_{a b} \bar{\nabla}_{a} \bar{E}_{+b}, & \Omega_{k i j} & =\frac{1}{8}\left(\sigma_{i j}\right)_{a b} \bar{\nabla}_{a} \bar{E}_{k b} .
\end{aligned}
$$


The non-zero components of $\bar{\Omega}$ are

$$
\begin{aligned}
& \bar{\Omega}_{++-}=-\frac{1}{2} \nabla_{a} E_{+a}, \quad \bar{\Omega}_{i+-}=-\frac{1}{2} \nabla_{a} E_{i a}, \quad \bar{\Omega}_{++i}=\frac{\mathrm{i}}{8}\left(\sigma_{i} \Pi\right)_{a \dot{a}} \bar{\nabla}_{\dot{a}} A_{a+}, \\
& \bar{\Omega}_{j+i}=\frac{\mathrm{i}}{8}\left(\sigma_{j} \Pi\right)_{a \dot{a}} \bar{\nabla}_{\dot{a}} A_{a i}, \quad \bar{\Omega}_{+-i}=\frac{1}{4}\left(\sigma_{i}\right)_{a \dot{a}} \nabla_{\dot{a}} E_{+a}, \quad \bar{\Omega}_{j-i}=\frac{1}{4}\left(\sigma_{i}\right)_{a \dot{a}} \nabla_{\dot{a}} E_{j a}, \\
& \bar{\Omega}_{+i j}=\frac{1}{8}\left(\sigma_{i j}\right)_{a b} \nabla_{a} E_{+b}, \quad \bar{\Omega}_{k i j}=\frac{1}{8}\left(\sigma_{i j}\right)_{a b} \nabla_{a} E_{k b} .
\end{aligned}
$$

The non-zero components of $P$ are

$$
P_{a b}=\frac{1}{2} \nabla_{-} W_{a b}, \quad P_{a \dot{a}}=-\frac{\mathrm{i}}{2} \bar{\nabla}_{\dot{a}} E_{+a}, \quad P_{\dot{a} a}=-\frac{\mathrm{i}}{2} \nabla_{\dot{a}} \bar{E}_{+a}, \quad P_{\dot{a} \dot{b}}=-\frac{\mathrm{i}}{2} \nabla_{\dot{a}} \bar{E}_{+\dot{b}} .
$$

The non-zero components of $C$ are

$$
\begin{aligned}
& C_{+-a}=-\frac{\mathrm{i}}{2} \nabla_{-} F_{a+-} \\
& C_{+-\dot{a}}=\frac{\mathrm{i}}{2} \nabla_{\dot{a}} \Omega_{++-}, \quad C_{+i a}=-\frac{\mathrm{i}}{2} \nabla_{-} F_{a+i}, \\
& C_{+i \dot{a}}=\frac{1}{2}\left(\sigma_{i} \Pi\right)_{a \dot{a}} \bar{E}_{+a}+\frac{\mathrm{i}}{2} \nabla_{\dot{a}} \Omega_{++i}, \quad C_{-i a}=-\frac{\mathrm{i}}{2} \nabla_{-} F_{a-i}, \quad C_{-i \dot{a}}=\frac{\mathrm{i}}{2} \nabla_{\dot{a}} \Omega_{+-i}, \\
& C_{i j a}=-\frac{\mathrm{i}}{4} \nabla_{-} F_{a i j}, \quad C_{i j \dot{a}}=\frac{\mathrm{i}}{2} \nabla_{\dot{a}} \Omega_{+i j} .
\end{aligned}
$$

The non-zero components of $\bar{C}$ are

$$
\begin{aligned}
& \bar{C}_{+-a}=\frac{\mathrm{i}}{2} \nabla_{-} F_{+-a}, \\
& \bar{C}_{+-\dot{a}}=\frac{\mathrm{i}}{2} \bar{\nabla}_{\dot{a}} \Omega_{++-}, \quad \bar{C}_{+i a}=\frac{\mathrm{i}}{2} \nabla_{-} F_{+i a} \\
& \bar{C}_{+i \dot{a}}=-\frac{1}{2}\left(\sigma_{i} \Pi\right)_{a \dot{a}} E_{+a}+\frac{\mathrm{i}}{2} \bar{\nabla}_{\dot{a}} \bar{\Omega}_{++i}, \\
& \bar{C}_{-i a}=\frac{\mathrm{i}}{2} \nabla_{-} F_{-i a}, \quad \bar{C}_{-i \dot{a}}=\frac{\mathrm{i}}{2} \bar{\nabla}_{\dot{a}} \bar{\Omega}_{+-i} \\
& \bar{C}_{i j a}=\frac{\mathrm{i}}{4} \nabla_{-} F_{i j a}, \\
& \bar{C}_{i j a}=\frac{\mathrm{i}}{2} \bar{\nabla}_{\dot{a}} \bar{\Omega}_{+i j} \text {. }
\end{aligned}
$$

Finally, the non.zero components of $R$ are

$$
\begin{aligned}
R_{+-+-} & =-\frac{1}{2} \nabla_{a} C_{+-a} \\
R_{+-+i} & =-\frac{\mathrm{i}}{2}\left(\sigma_{i} \Pi\right)_{a \dot{a}} P_{a \dot{a}}-\frac{1}{2} \nabla_{a} C_{+i a}, \\
R_{+i+-} & =-\frac{\mathrm{i}}{2}\left(\sigma_{i} \Pi\right)_{a \dot{a}} P_{\dot{a} a}-\frac{1}{2} \bar{\nabla}_{a} \bar{C}_{+i a}, \\
R_{+--i} & =-\frac{1}{2} \nabla_{a} C_{-i a} \\
R_{-i+-} & =-\frac{1}{2} \bar{\nabla}_{a} \bar{C}_{-i a}, \\
R_{+-i j} & =\frac{\mathrm{i}}{8}\left(\sigma_{[i} \Pi \sigma_{j]}\right)_{a b} P_{b a}-\frac{1}{2} \nabla_{a} C_{i j a} \\
R_{i j+-} & =\frac{\mathrm{i}}{8}\left(\sigma_{[i} \Pi \sigma_{j]}\right)_{a b} P_{a b}-\frac{1}{2} \bar{\nabla}_{a} \bar{C}_{i j a}, \\
R_{+i+j} & =\frac{1}{4}\left(\sigma_{i}\right)_{a \dot{a}} \nabla_{a} C_{+j \dot{a}}+\frac{\mathrm{i}}{8}\left(\sigma_{i} \Pi\right)_{a \dot{a}} \bar{\nabla}_{\dot{a}} F_{a+j}, \\
R_{+i-j} & =\frac{\mathrm{i}}{4}\left(\sigma_{i} \Pi \sigma_{j}\right)_{a b} P_{a b}+\frac{1}{4}\left(\sigma_{j}\right)_{a \dot{a}} \bar{\nabla}_{\dot{a}} \bar{C}_{+i a},
\end{aligned}
$$




$$
\begin{aligned}
R_{-j+i} & =\frac{\mathrm{i}}{4}\left(\sigma_{i} \Pi \sigma_{j}\right)_{a b} P_{b a}+\frac{1}{4}\left(\sigma_{j}\right)_{a \dot{a}} \nabla_{\dot{a}} C_{+i a}, \\
R_{+i j k} & =-\frac{\mathrm{i}}{8}\left(\sigma_{j k} \sigma_{i} \Pi\right)_{a \dot{a}} P_{\dot{a} a}+\frac{1}{8}\left(\sigma_{j k}\right)_{a b} \bar{\nabla}_{a} \bar{C}_{+i b}, \\
R_{j k+i} & =-\frac{\mathrm{i}}{8}\left(\sigma_{j k} \sigma_{i} \Pi\right)_{a \dot{a}} P_{a \dot{a}}+\frac{1}{8}\left(\sigma_{j k}\right)_{a b} \nabla_{a} C_{+i b}, \\
R_{-i-j} & =\frac{1}{4}\left(\sigma_{i}\right)_{a \dot{a}} \nabla_{\dot{a}} C_{-j a} \\
R_{-i j k} & =\frac{1}{4}\left(\sigma_{i}\right)_{a \dot{a}} \nabla_{\dot{a}} C_{j k a} \\
R_{j k-i} & =\frac{1}{4}\left(\sigma_{i}\right)_{a \dot{a}} \bar{\nabla}_{\dot{a}} \bar{C}_{j k a}, \\
R_{i j k l} & =-\frac{\mathrm{i}}{32}\left(\sigma_{[k} \Pi \sigma_{l]} \sigma_{i j}\right)_{a b} P_{b a}+\frac{1}{8}\left(\sigma_{i j}\right)_{a b} \nabla_{a} C_{k l b} .
\end{aligned}
$$

This completes the calculation of all superfields in the integrated vertex operator (6.26).

\section{Acknowledgments}

We would like to thank William Linch for useful discussions and comments on the draft. The work of BCV is partially supported by FONDECYT grant number 1151409 and CONICYT grant number DPI20140115.

\section{A Conventions}

We will use an $\mathfrak{s o}(4) \oplus \mathfrak{s o}(4)$ decomposition of the $\mathfrak{p s u}(2,2 \mid 4)$ algebra. The generators will be denoted by:

$$
\mathfrak{g}_{0}=\left(\mathrm{M}_{A B}, \mathrm{M}_{A}, \mathrm{M}_{I J}, \mathrm{M}_{I}\right), \quad \mathfrak{g}_{1}=\left(\mathrm{Q}_{a}, \mathrm{Q}_{\dot{a}}\right), \quad \mathfrak{g}_{2}=\left(\mathrm{T}, \mathrm{P}_{A}, \mathrm{~J}, \mathrm{P}_{I}\right), \quad \mathfrak{g}_{3}=\left(\overline{\mathrm{Q}}_{a}, \overline{\mathrm{Q}}_{\dot{a}}\right)
$$

Translations and supercharges $\left(\mathfrak{g}_{1}, \mathfrak{g}_{2}, \mathfrak{g}_{3}\right)$ will be hermitian and rotations $\mathfrak{g}_{0}$ will be antihermitian. This convention removes all i from the bosonic commutators. The non zero commutators are $\left(R\right.$ is the radius of $A d S_{5}$ and $\left.S^{5}\right)$ :

$$
\begin{aligned}
& {\left[\mathrm{M}_{A B}, \mathrm{M}_{C D}\right]=-\delta_{A[C} \mathrm{M}_{D] B}+\delta_{B[C} \mathrm{M}_{D] A}} \\
& {\left[\mathrm{M}_{A B}, \mathrm{M}_{C}\right]=\delta_{C[A} \mathrm{M}_{B]}, \quad\left[\mathrm{M}_{A}, \mathrm{M}_{B}\right]=-\mathrm{M}_{A B},} \\
& {\left[\mathrm{M}_{I J}, \mathrm{M}_{K L}\right]=-\delta_{I[K} \mathrm{M}_{L] J}+\delta_{J[K} \mathrm{M}_{L] I}, \quad\left[\mathrm{M}_{I J}, \mathrm{M}_{K}\right]=\delta_{K[I} \mathrm{M}_{J]}, \quad\left[\mathrm{M}_{I}, \mathrm{M}_{J}\right]=\mathrm{M}_{I J}} \\
& {\left[\mathrm{~T}, \mathrm{P}_{A}\right]=-\frac{1}{R^{2}} \mathrm{M}_{A}, \quad\left[\mathrm{P}_{A}, \mathrm{P}_{B}\right]=-\frac{1}{R^{2}} \mathrm{M}_{A B}, \quad\left[\mathrm{~J}, \mathrm{P}_{I}\right]=\frac{1}{R^{2}} \mathrm{M}_{I}, \quad\left[\mathrm{P}_{I}, \mathrm{P}_{J}\right]=\frac{1}{R^{2}} \mathrm{M}_{I J},} \\
& {\left[\mathrm{M}_{A B}, \mathrm{P}_{C}\right]=\delta_{C[A} \mathrm{P}_{B]}, \quad\left[\mathrm{M}_{A}, \mathrm{~T}\right]=-\mathrm{P}_{A}, \quad\left[\mathrm{M}_{A}, \mathrm{P}_{B}\right]=-\delta_{A B} \mathrm{~T}} \\
& {\left[\mathrm{M}_{I J}, \mathrm{P}_{K}\right]=\delta_{K[I} \mathrm{P}_{J]}, \quad\left[\mathrm{M}_{I}, \mathrm{~J}\right]=\mathrm{P}_{I}, \quad\left[\mathrm{M}_{I}, \mathrm{P}_{J}\right]=-\delta_{I J} J} \\
& {\left[\mathrm{M}_{A B}, \mathrm{Q}_{a}\right]=\frac{1}{2}\left(\sigma_{A B}\right)_{a b} \mathrm{Q}_{b}, \quad\left[\mathrm{M}_{A B}, \mathrm{Q}_{\dot{a}}\right]=\frac{1}{2}\left(\sigma_{A B}\right)_{\dot{a} \dot{b}} \mathrm{Q}_{\dot{b}}} \\
& {\left[\mathrm{M}_{A}, \mathrm{Q}_{a}\right]=-\frac{1}{2}\left(\sigma_{A}\right)_{a \dot{b}} \mathrm{Q}_{\dot{b}}, \quad\left[\mathrm{M}_{A}, \mathrm{Q}_{\dot{a}}\right]=-\frac{1}{2}\left(\sigma_{A}\right)_{\dot{a} b} \mathrm{Q}_{b}}
\end{aligned}
$$




$$
\begin{aligned}
& {\left[\mathrm{M}_{I J}, \mathrm{Q}_{a}\right]=\frac{1}{2}\left(\sigma_{I J}\right)_{a b} \mathrm{Q}_{b}, \quad\left[\mathrm{M}_{I J}, \mathrm{Q}_{\dot{a}}\right]=\frac{1}{2}\left(\sigma_{I J}\right)_{\dot{a} \dot{b}} \mathrm{Q}_{\dot{b}},} \\
& {\left[\mathrm{M}_{I}, \mathrm{Q}_{a}\right]=\frac{1}{2}\left(\sigma_{I}\right)_{a \dot{b}} \mathrm{Q}_{\dot{b}}, \quad\left[\mathrm{M}_{I}, \mathrm{Q}_{\dot{a}}\right]=-\frac{1}{2}\left(\sigma_{I}\right)_{\dot{a} b} \mathrm{Q}_{b},} \\
& {\left[\mathrm{M}_{A B}, \overline{\mathrm{Q}}_{a}\right]=\frac{1}{2}\left(\sigma_{A B}\right)_{a b} \overline{\mathrm{Q}}_{b}, \quad\left[\mathrm{M}_{A B}, \overline{\mathrm{Q}}_{\dot{a}}\right]=\frac{1}{2}\left(\sigma_{A B}\right)_{\dot{a} \dot{b}} \overline{\mathrm{Q}}_{\dot{b}},} \\
& {\left[\mathrm{M}_{A}, \overline{\mathrm{Q}}_{a}\right]=-\frac{1}{2}\left(\sigma_{A}\right)_{a \dot{b}} \overline{\mathrm{Q}}_{\dot{b}}, \quad\left[\mathrm{M}_{A}, \overline{\mathrm{Q}}_{\dot{a}}\right]=-\frac{1}{2}\left(\sigma_{A}\right)_{\dot{a} b} \overline{\mathrm{Q}}_{b},} \\
& {\left[\mathrm{M}_{I J}, \overline{\mathrm{Q}}_{a}\right]=\frac{1}{2}\left(\sigma_{I J}\right)_{a b} \overline{\mathrm{Q}}_{b}, \quad\left[\mathrm{M}_{I J}, \overline{\mathrm{Q}}_{\dot{a}}\right]=\frac{1}{2}\left(\sigma_{I J}\right)_{\dot{a} \dot{b}} \overline{\mathrm{Q}}_{\dot{b}},} \\
& {\left[\mathrm{M}_{I}, \overline{\mathrm{Q}}_{a}\right]=\frac{1}{2}\left(\sigma_{I}\right)_{a \dot{b}} \overline{\mathrm{Q}}_{\dot{b}}, \quad\left[\mathrm{M}_{I}, \overline{\mathrm{Q}}_{\dot{a}}\right]=-\frac{1}{2}\left(\sigma_{I}\right)_{\dot{a} b} \overline{\mathrm{Q}}_{b},} \\
& {\left[\mathrm{~T}, \mathrm{Q}_{a}\right]=-\left[\mathrm{J}, \mathrm{Q}_{a}\right]=\frac{\mathrm{i}}{2 R} \Pi_{a b} \overline{\mathrm{Q}}_{b}, \quad\left[\mathrm{~T}, \mathrm{Q}_{\dot{a}}\right]=\left[\mathrm{J}, \mathrm{Q}_{\dot{a}}\right]=\frac{\mathrm{i}}{2 R} \Pi_{\dot{a} \dot{b}} \overline{\mathrm{Q}}_{\dot{b}},} \\
& {\left[\mathrm{P}_{A}, \mathrm{Q}_{a}\right]=-\frac{\mathrm{i}}{2 R}\left(\sigma_{A}\right)_{a \dot{b}} \Pi_{\dot{b} \dot{c}} \overline{\mathrm{Q}}_{\dot{c}}, \quad\left[\mathrm{P}_{A}, \mathrm{Q}_{\dot{a}}\right]=-\frac{\mathrm{i}}{2 R}\left(\sigma_{A}\right)_{\dot{a} b} \Pi_{b c} \overline{\mathrm{Q}}_{c},} \\
& {\left[\mathrm{P}_{I}, \mathrm{Q}_{a}\right]=-\frac{\mathrm{i}}{2 R}\left(\sigma_{I}\right)_{a \dot{b}} \Pi_{\dot{b} \dot{c}} \overline{\mathrm{Q}}_{\dot{c}}, \quad\left[\mathrm{P}_{I}, \mathrm{Q}_{\dot{a}}\right]=-\frac{\mathrm{i}}{2 R}\left(\sigma_{I}\right)_{\dot{a} b} \Pi_{b c} \overline{\mathrm{Q}}_{c},} \\
& {\left[\mathrm{~T}, \overline{\mathrm{Q}}_{a}\right]=-\left[\mathrm{J}, \overline{\mathrm{Q}}_{a}\right]=-\frac{\mathrm{i}}{2 R} \Pi_{a b} \mathrm{Q}_{b}, \quad\left[\mathrm{~T}, \overline{\mathrm{Q}}_{\dot{a}}\right]=\left[\mathrm{J}, \overline{\mathrm{Q}}_{\dot{a}}\right]=-\frac{\mathrm{i}}{2 R} \Pi_{\dot{a} \dot{b}} \mathrm{Q}_{\dot{b}},} \\
& {\left[\mathrm{P}_{A}, \overline{\mathrm{Q}}_{a}\right]=\frac{\mathrm{i}}{2 R}\left(\sigma_{A}\right)_{a \dot{b}} \Pi_{\dot{b} \dot{c}} \mathrm{Q}_{\dot{c}}, \quad\left[\mathrm{P}_{A}, \overline{\mathrm{Q}}_{\dot{a}}\right]=\frac{\mathrm{i}}{2 R}\left(\sigma_{A}\right)_{\dot{a} b} \Pi_{b c} \mathrm{Q}_{c},} \\
& {\left[\mathrm{P}_{I}, \overline{\mathrm{Q}}_{a}\right]=\frac{\mathrm{i}}{2 R}\left(\sigma_{I}\right)_{a \dot{b}} \Pi_{\dot{b} \dot{c}} \mathrm{Q}_{\dot{c}}, \quad\left[\mathrm{P}_{I}, \overline{\mathrm{Q}}_{\dot{a}}\right]=\frac{\mathrm{i}}{2 R}\left(\sigma_{I}\right)_{\dot{a} b} \Pi_{b c} \mathrm{Q}_{c},} \\
& \left\{\mathrm{Q}_{a}, \mathrm{Q}_{b}\right\}=\delta_{a b}(\mathrm{~T}+\mathrm{J}), \quad\left\{\mathrm{Q}_{a}, \mathrm{Q}_{\dot{b}}\right\}=\sigma_{a \dot{b}}^{A} \mathrm{P}_{A}+\sigma_{a \dot{b}}^{I} \mathrm{P}_{I}, \quad\left\{\mathrm{Q}_{\dot{a}}, \mathrm{Q}_{\dot{b}}\right\}=\delta_{\dot{a} \dot{b}}(\mathrm{~T}-\mathrm{J}), \\
& \left\{\overline{\mathrm{Q}}_{a}, \overline{\mathrm{Q}}_{b}\right\}=\delta_{a b}(\mathrm{~T}+\mathrm{J}), \quad\left\{\overline{\mathrm{Q}}_{a}, \overline{\mathrm{Q}}_{\dot{b}}\right\}=\sigma_{a \dot{b}}^{A} \mathrm{P}_{A}+\sigma_{a \dot{b}}^{I} \mathrm{P}_{I}, \quad\left\{\overline{\mathrm{Q}}_{\dot{a}}, \overline{\mathrm{Q}}_{\dot{b}}\right\}=\delta_{\dot{a} \dot{b}}(\mathrm{~T}-\mathrm{J}), \\
& \left\{\mathrm{Q}_{a}, \overline{\mathrm{Q}}_{b}\right\}=-\frac{\mathrm{i}}{2 R}\left(\left(\sigma^{A B}\right)_{a c} \Pi_{c b} \mathrm{M}_{A B}-\left(\sigma^{I J}\right)_{a c} \Pi_{c b} \mathrm{M}_{I J}\right) \text {, } \\
& \left\{\mathrm{Q}_{a}, \overline{\mathrm{Q}}_{\dot{b}}\right\}=-\frac{\mathrm{i}}{R}\left(\left(\sigma^{A}\right)_{a \dot{c}} \Pi_{\dot{c} \dot{b}} \mathrm{M}_{A}-\left(\sigma^{I}\right)_{a \dot{c}} \Pi_{\dot{c} \dot{b}} \mathrm{M}_{I}\right) \text {, } \\
& \left\{\mathrm{Q}_{\dot{a}}, \overline{\mathrm{Q}}_{\dot{b}}\right\}=-\frac{\mathrm{i}}{2 R}\left(\left(\sigma^{A B}\right)_{\dot{a} \dot{c}} \Pi_{\dot{c} \dot{b}} \mathrm{M}_{A B}-\left(\sigma^{I J}\right)_{\dot{a} \dot{c}} \Pi_{\dot{c} \dot{b}} \mathrm{M}_{I J}\right), \\
& \left\{\mathrm{Q}_{\dot{a}}, \overline{\mathrm{Q}}_{b}\right\}=-\frac{\mathrm{i}}{R}\left(\left(\sigma^{A}\right)_{\dot{a} c} \Pi_{c b} \mathrm{M}_{A}+\left(\sigma^{I}\right)_{\dot{a} c} \Pi_{c b} \mathrm{M}_{I}\right) \text {. }
\end{aligned}
$$

\section{B Open and flat}

In this appendix we briefly review the construction of vertex operators in a light-cone gauge in a flat background $[20,29]$. We first start with an open pure spinor string in a flat background. The BRST gauge symmetry helps to fix the component $A_{\dot{a}}$ of $A_{\alpha}$ to zero.

We will see that the vertex operator is completely fixed by only the $\lambda^{\dot{a}} D_{\dot{a}}$ part of the BRST charge and the Virasoro constraint $k^{2}=0$. First we will use the frame in which the only non-vanishing component of the momentum is $k^{+}$, that is, the dependence on the space-time coordinates of all superfields is $e^{-2 \mathrm{i} k^{+} X^{-}}$. The equations coming form the complete BRST charge $Q U=0$, where $U=\lambda^{a} A_{a}$, are

$$
D_{\dot{a}} A_{b}=\sigma_{b \dot{a}}^{i} A_{i}, \quad D_{(a} A_{b)}=\delta_{a b} A_{+},
$$


where $D_{a}=\partial_{a}$ and $D_{\dot{a}}=\partial_{\dot{a}}-i k^{+} \theta^{\dot{a}}$. A fermionic superfield $W^{\alpha}$ can be defined such that the following equations are satisfied

$$
D_{a} A_{+}=0, \quad D_{a} A_{i}=\sigma_{a \dot{b}}^{i} W_{\dot{b}}, \quad D_{\dot{a}} A_{+}=-2 W_{\dot{a}}, \quad D_{\dot{a}} A_{i}=\sigma_{b \dot{a}}^{i} W_{b}, \quad W_{a}=-\mathrm{i} k^{+} A_{a} .
$$

The fermionic derivative of $W$ is related to $F=d A$, that is

$$
D_{a} W_{b}=-\frac{\mathrm{i}}{2} k^{+} \delta_{a b} A_{+}, \quad D_{\dot{a}} W_{b}=-\mathrm{i} k^{+} \sigma_{b \dot{a}}^{i} A_{i}, \quad D_{a} W_{\dot{b}}=0, \quad D_{\dot{a}} W_{\dot{b}}=\frac{\mathrm{i}}{2} k^{+} \delta_{\dot{a} \dot{b}} A_{+} .
$$

Because the last equation in (B.2), the second equation in (B.3) is trivially satisfied. Applying $D_{\dot{b}}$ to the last equation in (B.3) and using the third equation in (B.2) one obtains that $W_{\dot{a}}=0$ and, consequently, $A_{+}=0$. From now on, the only physical superfields are $A_{a}=\frac{\mathrm{i}}{k^{+}} W_{a}$ and $A_{i}$. Note that they depend on $\left(\theta^{\dot{a}}, X^{-}\right)$and satisfy the equations

$$
D_{\dot{a}} A_{b}=\sigma_{b \dot{a}}^{i} A_{i}, \quad D_{\dot{a}} A_{i}=-\mathrm{i} k^{+} \sigma_{b \dot{a}}^{i} A_{b} .
$$

The first equation completely fixes the physical vertex operator. The integrated vertex operator is

$$
\oint\left(\partial \theta^{a}-\mathrm{i} k^{+} d_{a}\right) A_{a}+\left(\Pi^{i}-2 \mathrm{i} k^{+} N^{-i}\right) A_{i}
$$

where $N^{-i}=\frac{1}{2} \sigma_{a \dot{b}}^{i} \lambda^{\dot{b}} \omega_{a}$.

Consider the closed string case. Using the BRST gauge symmetry, the unintegrated vertex operator is $U=\lambda^{a} \bar{\lambda}^{\bar{b}} A_{a \bar{b}}$. The equations from $Q U=0$ imply that $A_{a \bar{b}}$ is function of $\left(X^{-}, \theta^{\dot{a}}, \bar{\theta}^{\dot{\bar{a}}}\right)$ and it satisfies the equations

$$
D_{\dot{a}} A_{a \bar{b}}=\sigma_{a \dot{a}}^{i} A_{i \bar{b}}, \quad D_{\dot{a}} A_{i \bar{b}}=-\mathrm{i} k^{+} \sigma_{a \dot{a}}^{i} A_{a \bar{b}}, \quad D_{\dot{\bar{a}}} A_{a \bar{b}}=\sigma_{\bar{b} \dot{\bar{a}}}^{i} A_{a i}, \quad D_{\dot{\bar{a}}} A_{a i}=-\mathrm{i} k^{+} \sigma_{\bar{b} \dot{a}}^{i} A_{a \bar{b}} .
$$

The integrated vertex is $\int d^{2} z V$ and it satisfies $Q V=\partial \bar{W}-\bar{\partial} W$, where $Q W=\partial U, Q \bar{W}=$ $\bar{\partial} U$ and are given by

$$
\begin{aligned}
& W=\bar{\lambda}^{\bar{b}}\left(\left(\partial \theta^{a}-\mathrm{i} k^{+} d_{a}\right) A_{a \bar{b}}+\left(\Pi^{i}-2 \mathrm{i} k^{+} N^{-i}\right) A_{i \bar{b}}\right), \\
& \bar{W}=\lambda^{a}\left(\left(\bar{\partial} \bar{\theta}^{\bar{b}}-\mathrm{i} k^{+} \bar{d}_{\bar{b}}\right) A_{a \bar{b}}+\left(\bar{\Pi}^{i}-2 \mathrm{i} k^{+} \bar{N}^{-i}\right) A_{a i}\right),
\end{aligned}
$$

where

$$
N^{-i}=\frac{1}{2} \sigma_{a \dot{b}}^{i} \lambda^{\dot{b}} \omega_{a}, \quad \bar{N}^{-i}=\frac{1}{2} \sigma_{\bar{a} \dot{\bar{b}}}^{i} \bar{\lambda}^{\bar{b}} \bar{\omega}_{\bar{a}}
$$

The integrated vertex operator becomes

$$
\begin{aligned}
V= & \left(\partial \theta^{a} \bar{\partial} \bar{\theta}^{\bar{b}}-\mathrm{i} k^{+} d_{a} \bar{\partial} \bar{\theta}^{\bar{b}}-\mathrm{i} k^{+} \partial \theta^{a} \bar{d}_{\bar{b}}-\left(k^{+}\right)^{2} d_{a} \bar{d}_{\bar{b}}\right) A_{a \bar{b}} \\
& +\left(\partial \theta^{a} \bar{\Pi}^{i}-2 \mathrm{i} k^{+} \partial \theta^{a} \bar{N}^{-i}-\mathrm{i} k^{+} d_{a} \bar{\Pi}^{i}-2\left(k^{+}\right)^{2} d_{a} \bar{N}^{-i}\right) A_{a i} \\
& -\left(\Pi^{i} \bar{\partial} \bar{\theta}^{\bar{a}}-2 \mathrm{i} k^{+} N^{-i} \bar{\partial} \bar{\theta}^{\bar{a}}-\mathrm{i} k^{+} \Pi^{i} \bar{d}_{\bar{a}}-2\left(k^{+}\right)^{2} N^{-i} \bar{d}_{\bar{a}}\right) A_{i \bar{a}} \\
& +\left(\Pi^{i} \bar{\Pi}^{j}-2 \mathrm{i} k^{+} \Pi^{j} \bar{N}^{-i}-2 \mathrm{i} k^{+} N^{-j} \bar{\Pi}^{i}+8\left(k^{+}\right)^{2} N^{-i} \bar{N}^{-j}\right) A_{i j}
\end{aligned}
$$

where

$$
A_{i j}=\frac{1}{64} \sigma_{i}^{a \dot{b}} \sigma_{j}^{\bar{a} \overline{\bar{b}}} D_{\dot{b}} D_{\dot{\bar{b}}} A_{a \bar{a}}
$$


Open Access. This article is distributed under the terms of the Creative Commons Attribution License (CC-BY 4.0), which permits any use, distribution and reproduction in any medium, provided the original author(s) and source are credited.

\section{References}

[1] N. Beisert et al., Review of AdS/CFT Integrability: An Overview, Lett. Math. Phys. 99 (2012) 3 [arXiv: 1012.3982] [INSPIRE].

[2] G. Arutyunov and S. Frolov, Foundations of the AdS $S_{5} \times S^{5}$ Superstring. Part I, J. Phys. A 42 (2009) 254003 [arXiv:0901.4937] [INSPIRE].

[3] O.A. Bedoya, L.I. Bevilaqua, A. Mikhailov and V.O. Rivelles, Notes on $\beta$-deformations of the pure spinor superstring in $A d S_{5} \times S^{5}$, Nucl. Phys. B 848 (2011) 155 [arXiv: 1005.0049] [INSPIRE].

[4] A. Mikhailov, Finite dimensional vertex, JHEP 12 (2011) 005 [arXiv:1105.2231] [INSPIRE].

[5] N. Berkovits and T. Fleury, Harmonic Superspace from the $A d S_{5} \times S^{5}$ Pure Spinor Formalism, JHEP 03 (2013) 022 [arXiv:1212.3296] [INSPIRE].

[6] B.C. Vallilo and L. Mazzucato, The Konishi multiplet at strong coupling, JHEP 12 (2011) 029 [arXiv: 1102.1219] [INSPIRE].

[7] M. Heinze, Spectrum and Quantum Symmetries of the AdS $S_{5} \times S^{5}$ Superstring, Ph.D. thesis, Humboldt U., Berlin, 2014. arXiv:1507.03005 [INSPIRE].

[8] B.C. Vallilo, Flat currents in the classical $A d S_{5} \times S^{5}$ pure spinor superstring, JHEP 03 (2004) 037 [hep-th/0307018] [INSPIRE].

[9] O. Chandía, W.D. Linch and B.C. Vallilo, Master symmetry in the $A d S_{5} \times S^{5}$ pure spinor string, JHEP 01 (2017) 024 [arXiv: 1607.00391] [INSPIRE].

[10] A. Mikhailov and S. Schäfer-Nameki, Algebra of transfer-matrices and Yang-Baxter equations on the string worldsheet in $A d S_{5} \times S^{5}$, Nucl. Phys. B 802 (2008) 1 [arXiv:0712.4278] [INSPIRE].

[11] R. Benichou, First-principles derivation of the AdS/CFT Y-systems, JHEP 10 (2011) 112 [arXiv:1108.4927] [INSPIRE].

[12] S.K. Ashok, R. Benichou and J. Troost, Conformal Current Algebra in Two Dimensions, JHEP 06 (2009) 017 [arXiv: 0903.4277] [INSPIRE].

[13] R. Benichou and J. Troost, The Conformal Current Algebra on Supergroups with Applications to the Spectrum and Integrability, JHEP 04 (2010) 121 [arXiv:1002.3712] [INSPIRE].

[14] L. Eberhardt and K. Ferreira, The plane-wave spectrum from the worldsheet, arXiv: 1805.12155 [INSPIRE].

[15] D.E. Berenstein, J.M. Maldacena and H.S. Nastase, Strings in flat space and pp waves from $N=4$ superYang-Mills, JHEP 04 (2002) 013 [hep-th/0202021] [INSPIRE].

[16] R.R. Metsaev, Type IIB Green-Schwarz superstring in plane wave Ramond-Ramond background, Nucl. Phys. B 625 (2002) 70 [hep-th/0112044] [INSPIRE].

[17] R.R. Metsaev and A.A. Tseytlin, Exactly solvable model of superstring in Ramond-Ramond plane wave background, Phys. Rev. D 65 (2002) 126004 [hep-th/0202109] [INSPIRE]. 
[18] N. Berkovits, Conformal field theory for the superstring in a Ramond-Ramond plane wave background, JHEP 04 (2002) 037 [hep-th/0203248] [INSPIRE].

[19] O. Chandía, L. Ibiapina Bevilaqua and B.C. Vallilo, AdS pure spinor superstring in constant backgrounds, JHEP 06 (2014) 029 [arXiv:1404.0974] [INSPIRE].

[20] R.L. Jusinskas, Spectrum generating algebra for the pure spinor superstring, JHEP 10 (2014) 22 [arXiv: 1406.1902] [INSPIRE].

[21] M. Hatsuda, K. Kamimura and M. Sakaguchi, Super-P P wave algebra from super-AdS $\times S$ algebras in eleven-dimensions, Nucl. Phys. B 637 (2002) 168 [hep-th/0204002] [InSPIRE].

[22] M. Blau, J.M. Figueroa-O'Farrill, C. Hull and G. Papadopoulos, A new maximally supersymmetric background of IIB superstring theory, JHEP 01 (2002) 047 [hep-th/0110242] [INSPIRE].

[23] M. Blau, J.M. Figueroa-O'Farrill, C. Hull and G. Papadopoulos, Penrose limits and maximal supersymmetry, Class. Quant. Grav. 19 (2002) L87 [hep-th/0201081] [INSPIRE].

[24] M. Günaydin and N. Marcus, The Spectrum of the $S^{5}$ Compactification of the Chiral $N=2$, $D=10$ Supergravity and the Unitary Supermultiplets of U(2,2/4), Class. Quant. Grav. 2 (1985) L11 [INSPIRE].

[25] N. Berkovits, M. Bershadsky, T. Hauer, S. Zhukov and B. Zwiebach, Superstring theory on $A d S_{2} \times S^{2}$ as a coset supermanifold, Nucl. Phys. B 567 (2000) 61 [hep-th/9907200] [INSPIRE].

[26] P.A. Grassi and L. Tamassia, Vertex operators for closed superstrings, JHEP 07 (2004) 071 [hep-th/0405072] [INSPIRE].

[27] A. Mikhailov, Symmetries of massless vertex operators in $A d S_{5} \times S^{5}$, J. Geom. Phys. 62 (2012) 479 [arXiv:0903.5022] [INSPIRE].

[28] L. Brink, M.B. Green and J.H. Schwarz, Ten-dimensional Supersymmetric Yang-Mills Theory With SO(8) - Covariant Light Cone Superfields, Nucl. Phys. B 223 (1983) 125 [InSPIRE].

[29] N. Berkovits and R. Lipinski Jusinskas, Light-Cone Analysis of the Pure Spinor Formalism for the Superstring, JHEP 08 (2014) 102 [arXiv:1406.2290] [INSPIRE].

[30] N. Berkovits, Simplifying and Extending the $A d S_{5} \times S^{5}$ Pure Spinor Formalism, JHEP 09 (2009) 051 [arXiv:0812.5074] [INSPIRE].

[31] O. Chandía and B.C. Vallilo, A superfield realization of the integrated vertex operator in an $A d S_{5} \times S^{5}$ background, JHEP 10 (2017) 178 [arXiv:1709.05517] [INSPIRE]. 